ARTICLE

\title{
Structural basis for active single and double ring complexes in human mitochondrial Hsp60-Hsp10 chaperonin
}

\author{
Yacob Gomez-Llorente ${ }^{1,5}$, Fady Jebara ${ }^{2,5}$, Malay Patra ${ }^{2,5}$, Radhika Malik1', Shahar Nisemblat ${ }^{2}$, \\ Orna Chomsky-Hecht ${ }^{2}$, Avital Parnas ${ }^{2}$, Abdussalam Azem (1) ${ }^{2 凶}$, Joel A. Hirsch (1) ${ }^{2 \otimes} \&$ \\ Iban Ubarretxena-Belandia (iD) 1,3,4凶
}

$\mathrm{mHsp60-mHsp10} \mathrm{assists} \mathrm{the} \mathrm{folding} \mathrm{of} \mathrm{mitochondrial} \mathrm{matrix} \mathrm{proteins} \mathrm{without} \mathrm{the} \mathrm{negative}$ ATP binding inter-ring cooperativity of GroEL-GroES. Here we report the crystal structure of an ATP (ADP:BeF ${ }_{3}$-bound) ground-state mimic double-ring $\left.\mathrm{mHsp} \mathrm{O}_{14}-(\mathrm{mHsp10})_{7}\right)_{2}$ football complex, and the cryo-EM structures of the ADP-bound successor mHsp60 ${ }_{14}-\left(\mathrm{mHsp10}_{7}\right)_{2}$ complex, and a single-ring $\mathrm{mHsp60}_{7}-\mathrm{mHsp}_{7}$ half-football. The structures explain the nucleotide dependence of $\mathrm{mHsp60}$ ring formation, and reveal an inter-ring nucleotide symmetry consistent with the absence of negative cooperativity. In the ground-state a two-fold symmetric $\mathrm{H}$-bond and a salt bridge stitch the double-rings together, whereas only the $\mathrm{H}$-bond remains as the equatorial gap increases in an ADP football poised to split into halffootballs. Refolding assays demonstrate obligate single- and double-ring $\mathrm{mHsp} 60$ variants are active, and complementation analysis in bacteria shows the single-ring variant is as efficient as wild-type $\mathrm{mHsp60}$. Our work provides a structural basis for active single- and double-ring complexes coexisting in the $\mathrm{mHsp60-mHsp10} \mathrm{chaperonin} \mathrm{reaction} \mathrm{cycle.}$

\footnotetext{
${ }^{1}$ Department of Pharmacological Sciences, Icahn School of Medicine at Mount Sinai, New York, NY 10029, USA. ${ }^{2}$ Department of Biochemistry and Molecular Biology, School of Neurobiology, Biochemistry and Biophysics, The George S. Wise Faculty of Life Sciences, Tel Aviv University, Tel Aviv, Israel. ${ }^{3}$ Instituto Biofisika (UPV/EHU, CSIC), University of the Basque Country, E-48940 Leioa, Spain. ${ }^{4}$ Ikerbasque, Basque Foundation for Science, 48013 Bilbao, Spain. ${ }^{5}$ These authors

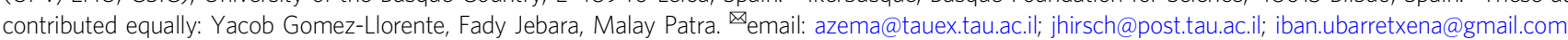


T he group I mitochondrial chaperonin $\mathrm{mHsp} 60$ and its cochaperonin mHsp10 assist the folding of mitochondrialimported proteins, and correct misfolded polypeptides resulting from mitochondrial stress ${ }^{1}$. Deletion of the $\mathrm{mHsp} 60$ gene in yeast is lethal ${ }^{2}$, and inactivation of its homologue in mice leads to embryonic mortality ${ }^{3}$. In addition to their primary protein-folding activity in the matrix, human $\mathrm{mHsp} 60$ and $\mathrm{mHsp} 10$ have also been implicated in a wide range of extra-mitochondrial and cytosolic activities, including the regulation of inflammatory cytokines, apoptopic processes, and carcinogenesis ${ }^{4-10}$. Several genetic neurodegenerative disorders have also been associated with defects in the human $\mathrm{mHsp} 60-\mathrm{mHsp} 10$ chaperonin system ${ }^{11-14}$.

Human mHsp60 shares $\sim 51 \%$ amino acid identity (Supplementary Fig. 1) with the best-studied group I chaperonin, GroEL from E. coli ${ }^{15,16}$. Like GroEL, human mHsp60 can also assemble into stacked back-to-back double-heptameric rings capped by a dome-shaped heptameric mHsp10 (GroES in the case of E. coli) co-chaperonin lid to form ATP-driven nanocages for a substrate-protein to fold in confinement ${ }^{17,18}$. Despite the high sequence homology, mHsp60 displays notable differences with GroEL in its mechanism of assembly and protein-folding reaction cycle. Whereas GroEL assembles into tight double-heptameric rings even in the absence of nucleotide ${ }^{19,20}$, oligomerization and double-ring formation for mHsp60 depends on $\mathrm{ATP}^{21}$. Negative ATP-binding inter-ring cooperativity ${ }^{22}$ characterizes the reaction cycle in GroEL, so that when the trans-ring in GroEL binds ATP the cis-ring does not. Accordingly, the canonical model for GroEL posits the reaction cycle begins when the GroEL double ring engages the lid formed by GroES, capping one end of the barrel at a time, and where the predominant functional complex is the socalled asymmetric bullet $\mathrm{GroEL}_{14}-\mathrm{GroES}_{7}{ }^{17,18}$. In this model, alternation between two forms of bullet complexes, the ADP and the ATP form, characterize the reaction cycle. An alternative model argues that in the presence of substrate-protein the protein-folding cycle also includes the so-called American football GroEL ${ }_{14}-\left(\mathrm{GroES}_{7}\right)_{2}$ complexes $^{23-25}$. In this latter model, the reaction cycle comprises alternating double-ring football and bullet complexes.

In contrast to GroEL, mHsp60 does not display negative ATPbinding inter-ring cooperativity at any point in its reaction cycle $^{21}$. In addition, in the presence of mHsp10 and ATP both single- and double-ring assemblies have been observed ${ }^{21,26}$. Consequently, we postulated a protein-folding reaction cycle devoid of negative cooperativity (Fig. 1a), where both football $\mathrm{mHsp}_{14}-\left(\mathrm{mHsp} 10_{7}\right)_{2}$ and half-football $\mathrm{mHsp} 0_{7}-\mathrm{mHsp} 10_{7}$ complexes coexist ${ }^{21,27}$. However, detailed structural evidence for this model was lacking.

We have recently solved the crystal structure (PDB 4PJ1) of a human $\mathrm{mHsp60} \mathrm{E}^{\mathrm{3} 21 \mathrm{~K}_{14}-\left(\mathrm{mHsp} 10_{7}\right)_{2}}$ variant in the form of a football complex bound to ADP at $3.15 \AA$ resolution ${ }^{28}$. With all mHsp60 subunits in both rings occupied by ADP, we suggested this structure corresponded to a post ATP hydrolysis intermediate representing the complex at an early stage of dissociation. Although this variant cannot progress through the folding cycle, as it is unable to release mHsp10 and liberate the folded substrate-protein ${ }^{29}$, it provided structural evidence for the formation of mHsp60-mHsp10 football complexes.

Here we employ both X-ray crystallography and cryo-electron microscopy (cryo-EM) to solve structures of wild-type (WT) $\mathrm{mHsp} 60-\mathrm{mHsp} 10$ complexes representing a ground-state particle ready for folding, the $\mathrm{ADP}: \mathrm{BeF}_{3}$-bound double-ring $\mathrm{mHsp} 60_{14^{-}}$ $\left(\mathrm{mHsp} 10_{7}\right)_{2}$ football, its apparent successor state, an ADP-bound mHsp60 $0_{14}-\left(\mathrm{mHsp} 10_{7}\right)_{2}$ football, and an ADP-bound single-ring $\mathrm{mHsp} 60_{7}-\mathrm{mHsp} 10_{7}$ half-football, at resolutions ranging from 3.8 to $3.1 \AA$. Complemented with in vitro and in vivo assays with obligate double and single-ring $\mathrm{mHsp} 60$ variants, our work provides a structural basis for active football and half-football complexes coexisting in the reaction cycle of human mHsp60-mHsp10.

\section{Results}

Structures of $\mathrm{mHsp60}-\mathrm{mHsp} 10$ reaction cycle intermediates. For cryo-EM we employed functional WT human mHsp60 and mHsp10. Mixing purified mHsp60 and mHsp10 subunits (protomers) at a 1:1.2 molar ratio in the presence of ATP, followed immediately by adsorption onto homemade lacey gold coated grids and vitrification, allowed us to record cryo-EM movies of mHsp60-mHsp10 complexes (Supplementary Fig. 2a) with a detectable signal close to $3.0 \AA$ (Supplementary Fig. 2b). Twodimensional (2D) classification revealed that mHsp60 and mHsp10 assembled primarily into football complexes (Fig. 1b) accounting for $\sim 70 \%$ of the particles, followed by half-football complexes ( $\sim 30 \%$ of the particles) (Supplementary Fig. $2 b)$. These mHsp60-mHsp10 assemblies have been observed before in the presence of $\mathrm{ATP}^{21}$. In agreement with previous $\mathrm{EM}^{26}$ and biochemical $^{21}$ data, bullet mHsp60-mHsp10 complexes were not observed in any of our images (Supplementary Fig. 2a). Upon image processing and after imposing D7 symmetry, we obtained a three-dimensional (3D) reconstruction (Supplementary Fig. 3; Table 1 displays structure determination statistics) of the mHsp60-mHsp10 football complexes at $3.08 \AA$ resolution (EMD9195), based on the gold-standard FSC $=0.143$ criterion (Supplementary Fig. 4a ${ }^{30}$. The refined model (Fig. 1d; PDB 6MRC) fits the cryo-EM density map well (Supplementary Fig. 5a, b), where side-chain densities for most amino acid residues of the mHsp60 and mHsp10 subunits were clearly resolved (Supplementary Fig. 5c). Independently of the football complexes, we also determined a $3.83 \AA$ resolution C7 symmetry $3 \mathrm{D}$ reconstruction of the half-football complexes observed in this data set (EMD-9196; Supplementary Figs. 4c, 6). The refined model of the half-football is shown in Fig. 1e (PDB 6MRD).

Biochemical experiments showed that addition of ATP and $\mathrm{BeF}_{3}$ generated a stable mHsp60-mHsp10 football complex unable to progress through the reaction cycle and assist substrate-protein folding (Supplementary Fig. 7a). In solution in the absence of ATP size-exclusion chromatography coupled with multi-angle light-scattering (SEC-MALS) showed WT human mHsp60 eluted (Supplementary Fig. 7b, d) mainly as monomers and single heptameric rings (apparent molecular weight of $\sim 445 \mathrm{kDa}$ ). In contrast, under the same conditions GroEL eluted (Supplementary Fig. 7b, d) as double-heptameric rings (apparent molecular weight of $\sim 792 \mathrm{kDa}$ ). In addition, mHsp10 eluted (Supplementary Fig. 7b, d) as single heptameric rings (apparent molecular weight of $\sim 75 \mathrm{kDa}$ ). In the presence of ATP, mHsp10 and mHsp60 eluted as single-ring complexes (Supplementary Fig. 7c, d). However, in the presence of ATP and $\mathrm{BeF}_{3}$, which upon ATP hydrolysis yield the ATP ground-state mimic ADP:BeF $3, \mathrm{mHsp}_{10}$ and mHsp60 eluted (Supplementary Fig. $7 \mathrm{c}, \mathrm{d}$ ) as oligomers with an apparent molecular weight of $\sim 823 \mathrm{kDa}$, consistent with a stable double-heptameric ring, likely a football complex. Thus, to obtain the structure of a ground-state mHsp60-mHsp10 football complex, we crystallized WT human mHsp60 and mHsp10 in the presence of ATP and $\mathrm{BeF}_{3}$. A diffraction data set with Bragg limits of $3.7 \AA$ enabled us to solve the structure (Fig. 1c; PDB 6HT7) using molecular replacement with a search model based on the $3.08 \AA$ refined cryo-EM structure (Table 2 displays crystallographic statistics).

The near-atomic resolution structures of these three assemblies are depicted in Fig. 1c-e in the order of the postulated reaction cycle (Fig. 1a), i.e., stages III through V. In the two football complexes, their respective football halves we term the north pole 
a

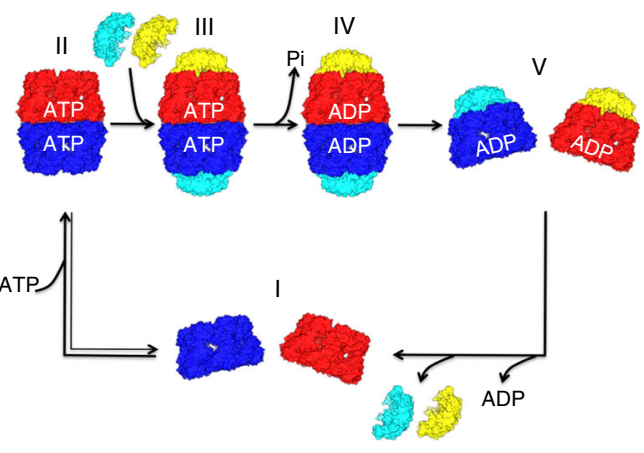

b

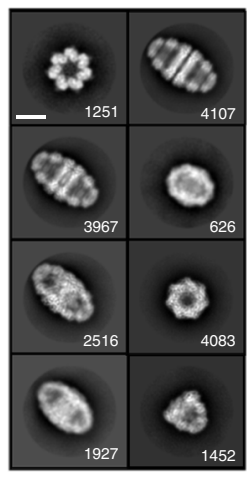

C

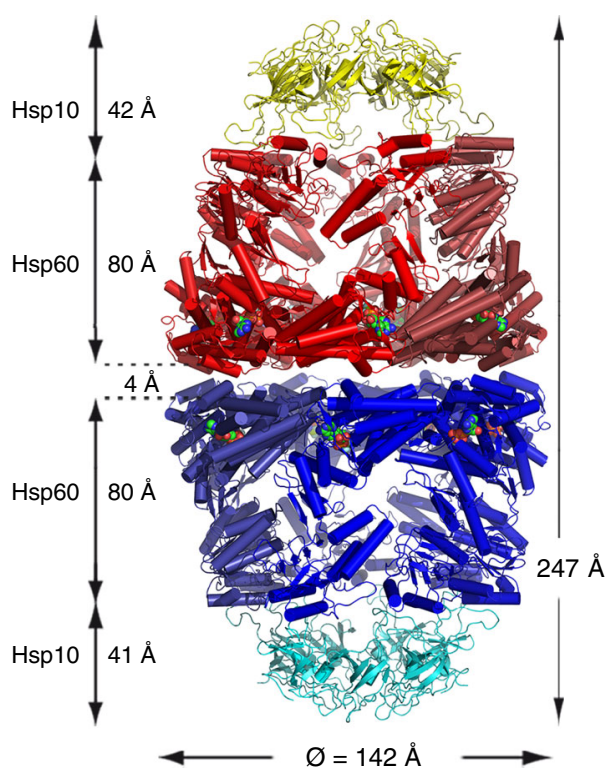

$-\frac{A}{4} 90^{\circ}$

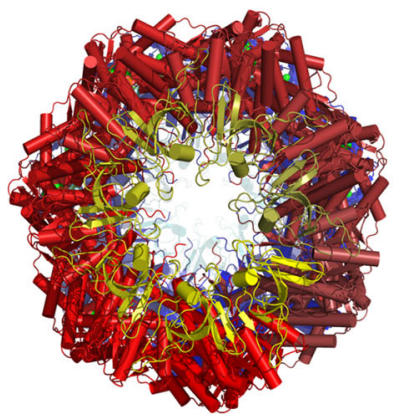

d
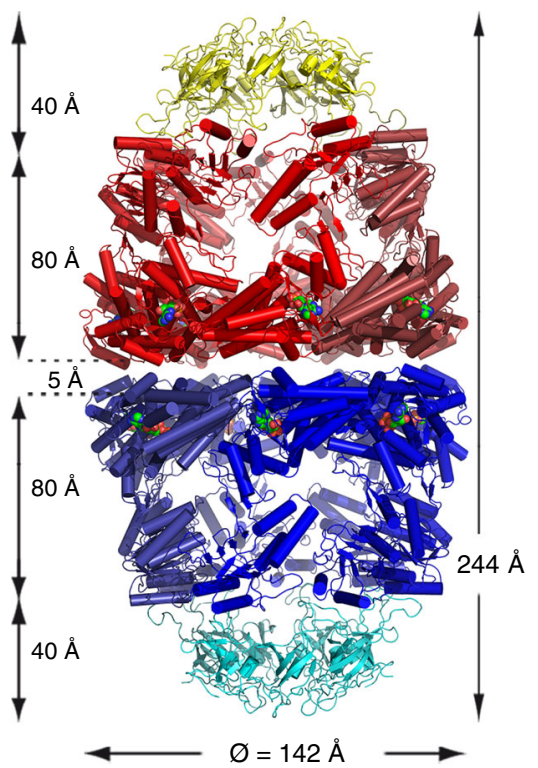

$f 90^{\circ}$

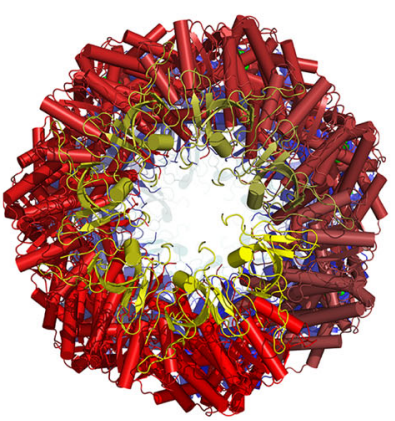

e

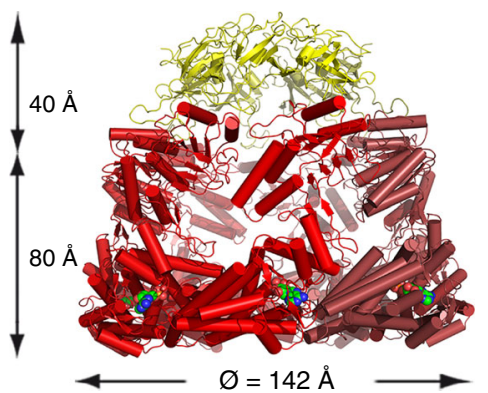

Fig. 1 Structures of mHsp60-mHsp10 complexes. a Published model for the reaction cycle of mHsp60-mHsp10 (adapted from ref. ${ }^{27}$ ). Red and blue denote $\mathrm{mHsp} 60$ heptameric north and south rings, respectively. Yellow and cyan denote $\mathrm{mHsp10}$ heptameric north and south lids, respectively. The same color scheme applies to the remaining figure panels. Nucleotide states are labeled. b Selected reference-free 2D class averages of $\mathrm{mHsp} 60-\mathrm{mH}$ sp10 complexes show detailed structural features. Side views clearly identify football and half-football complexes. In the classes representing front views, the two complexes can be distinguished by taking into account that the footballs have D7 symmetry (top class left column) and the half-footballs C7 symmetry (third class right column). The numbers show the particles per class, and the scale bar represents $100 \AA$. c Cartoon depiction of the ADP:BeF 3 bound $\mathrm{mHsp60}_{14}-\left(\mathrm{mHsp10}_{7}\right)_{2}$ football assembly derived from the $3.7 \AA$ resolution crystal structure viewed down a twofold dyad orthogonal to the sevenfold molecular axis. Below is a top view down the sevenfold axis. Individual $\mathrm{mHsp60}$ subunits are colored in graded hues of the relevant color. Spheres depict the nucleotide. $\mathbf{d}$ Cartoon depiction of the ADP-bound $\mathrm{mHsp60}_{14}-\left(\mathrm{mHsp}_{10}\right)_{2}$ football assembly derived from the $3.08 \AA$ resolution cryo-EM structure.

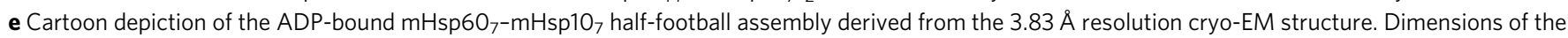
complexes are, respectively, indicated, using symmetrical $\mathrm{C} \alpha$ atoms to define limiting planes. 


\begin{tabular}{|c|c|c|}
\hline & $\begin{array}{l}\text { ADP football } \\
\text { (EMDB-9195) } \\
\text { (PDB 6MRC) }\end{array}$ & $\begin{array}{l}\text { ADP half-football } \\
\text { (EMDB-9196) } \\
\text { (PDB 6MRD) }\end{array}$ \\
\hline \multicolumn{3}{|l|}{$\begin{array}{l}\text { Data collection and } \\
\text { processing }\end{array}$} \\
\hline Magnification & 22,500 & 22,500 \\
\hline Voltage (kV) & 300 & 300 \\
\hline Electron exposure $\left(e-/ \AA^{2}\right)$ & 63 & 63 \\
\hline Defocus range $(\mu \mathrm{m})$ & $0.8-2.5$ & $0.8-2.5$ \\
\hline Pixel size $(\AA)$ & 1.07 & 1.07 \\
\hline Symmetry imposed & D7 & $\mathrm{C7}$ \\
\hline Initial particle images (no.) & 162,669 & 162,669 \\
\hline Final particle images (no.) & 66,013 & 10,972 \\
\hline Map resolution $(\AA)$ & 3.08 & 3.83 \\
\hline FSC threshold & 0.143 & 0.143 \\
\hline \multicolumn{3}{|l|}{ Refinement } \\
\hline Initial model used (PDB code) & 4PJ1 & $6 \mathrm{MRC}$ \\
\hline Model resolution $(\AA)$ & 3.08 & 3.83 \\
\hline FSC threshold & 0.143 & 0.143 \\
\hline Map sharpening $B$ factor $\left(\AA^{2}\right)$ & 140.8 & 111.6 \\
\hline \multicolumn{3}{|l|}{ Model composition } \\
\hline Non-hydrogen atoms & 66,066 & 33,033 \\
\hline Protein residues & 8792 & 4396 \\
\hline Ligands & 14 & 7 \\
\hline \multicolumn{3}{|l|}{$B$ factors $\left(\AA^{2}\right)$} \\
\hline Protein & 84.6 & 153.9 \\
\hline Ligand & 56.9 & 125.6 \\
\hline \multicolumn{3}{|l|}{ R.m.s. deviations } \\
\hline Bond lengths $(\AA)$ & 0.010 & 0.005 \\
\hline Bond angles $\left(^{\circ}\right)$ & 1.25 & 1.05 \\
\hline \multicolumn{3}{|l|}{ Validation } \\
\hline MolProbity score & 2.48 & 2.69 \\
\hline Clashscore & 27.46 & 44.83 \\
\hline Poor rotamers $(\%)$ & 0 & 0 \\
\hline EMRinger score & 3.46 & 0.97 \\
\hline \multicolumn{3}{|l|}{ Ramachandran plot } \\
\hline Favored (\%) & 89.69 & 89.77 \\
\hline Allowed (\%) & 9.84 & 10.07 \\
\hline Disallowed (\%) & 0.47 & 0.16 \\
\hline
\end{tabular}

and south pole. Features shared by all three structures include mHsp60 protomers in an extended conformation along the molecular symmetry axis with clearly defined domains, namely an equatorial ATP-binding domain (residues 1-137, 411-526), an intermediate hinge domain (residues 138-191, 375-411), and an apical domain (residues 192-374) (Supplementary Fig. 5b). All $\mathrm{mHsp} 60$ rings are bound to $\mathrm{mHsp} 10$ lids, where $\mathrm{mHsp} 10$ appears in the obligate heptameric form ${ }^{31}$ (Supplementary Fig. 7b, d), with little conformational variation between subunits (RMSD < $0.3 \AA$ A : Supplementary Table 1). Each mHsp10 protomer adopts the canonical seven-strand $\beta$-barrel structure and exposes a flexible loop sequence of twenty residues (mobile loop) that mediates the interaction with helices $\mathrm{H}$ and I of the mHsp60 apical domains. This $\mathrm{mHsp} 10$ lid conformation is rigid with low RMSD superposition values between all structures $(\mathrm{RMSD}<0.5$ Å; Supplementary Table 2).

Density features in the nucleotide-binding sites allowed us to unambiguously identify the nucleotide state of all $\mathrm{mHsp} 60-\mathrm{mHsp} 10$ complexes (Fig. 2). For the ground-state football crystal structure (stage III, Fig. 1c), we modeled $\mathrm{ADP}: \mathrm{BeF}_{3}, \mathrm{Mg}^{2+}$ and $\mathrm{K}^{+}$only at later stages of refinement where the density was most consistent with such an interpretation. $F o-F c$ difference maps display strong positive peaks when ADP alone is modeled. At the current resolution, it is not possible to precisely place atoms based on the
Table 2 Crystallographic data collection and refinement statistics.

Data collection

Wavelength $0.976 \AA$

Resolution range 48.95-3.7 (3.832-3.7)

$\begin{array}{ll}\text { Space group } & \text { P } 212121\end{array}$

Cell dimensions

$a, b, c(\AA)$

$\alpha, \beta, \gamma\left({ }^{\circ}\right)$

Total reflections

Unique reflections

Completeness (\%)

Redundancy

I/ $\sigma$

Wilson B factor

R-merge

$\mathrm{CC} 1 / 2$

$141.6,295.8,326.6$

$90^{\circ}, 90^{\circ}, 90^{\circ}$

607,096

144,762

$98.7 \%(98.1 \%)$

4.2

$6.95(1.45)$

120.91

$14.3 \%$

99.7

Refinement

Reflections used in 144523 (14233)

refinement

Reflections used for R-free 1998 (197)

R-work $\quad 0.2421(0.3472)$

R-free

$0.2905(0.3665)$

Number of atoms

Macromolecules $\quad 65,568$

Ligands $\quad 462$

Protein residues $\quad 8791$

RMSD

Bonds $(\AA) \quad 0.009$

Angles (degrees) $\quad 0.98$

Average $B$ factor $\left(\AA^{2}\right)$

Macromolecules $\quad 146.67$

Ligands $\quad 111.59$

Statistics for the highest-resolution shell are shown in parentheses.

electron density features but the density for the pocket, also seen with omit maps (Fig. 2a), support our modeling of the $\mathrm{BeF}_{3}, \mathrm{Mg}^{2+}$, and $\mathrm{K}^{+}$moieties. All nucleotide pockets in this complex appear alike, and we modeled them all as occupied by $\mathrm{ADP}: \mathrm{BeF}_{3}$. For the ADP football (stage IV, Fig. 1d), we conclude with confidence, as a result of the high resolution and quality of our cryo-EM map, that the nucleotide-binding sites in all fourteen $\mathrm{mHsp} 60$ subunits in both symmetric halves of the football are occupied by ADP and $\mathrm{Mg}^{2+}$, with no evidence for the presence of $\gamma$ phosphate or $\mathrm{K}^{+}$(Fig. $2 \mathrm{~b}$ ). The specific amino acid interactions with ADP and $\mathrm{Mg}^{2+}$ mirror those reported in the ADP-bound crystal mHsp60 $0^{\mathrm{E} 321 \mathrm{~K}}-\mathrm{mHsp} 10$ structure $^{28}$, except that in our map several water molecules are also visible. The same ADP-bound nucleotide state (Fig. 2c) is found in the half-football (stage V, Fig. 1e).

Intra-ring subunit interface. mHsp60 exhibits an ATPdependent monomer/heptamer/tetradecamer oligomeric equilibrium in a protein concentration-dependent manner ${ }^{21}$. What is the structural basis for this equilibrium? One region, structurally conserved in all three current structures, is the equatorial domain $\beta$-sheet formed from the $\mathrm{N}$-terminal and $\mathrm{C}$-terminal $\beta$-strands of one subunit, and a $\beta$-hairpin from the neighboring subunit (Supplementary Fig. 8a). This area, stabilized by both main chain and side-chain H-bonds and VDW interactions, is proximal to the nucleotide-binding pocket and the position of the $\gamma$ phosphate, suggesting a structural means by which ATP binding might assist ring formation ${ }^{21}$. Consistent with this structural observation, a three-residue $\mathrm{N}$-terminal truncation abrogates the need for nucleotide in ring formation while a pathogenic point mutant D3G destabilizes it ${ }^{32}$. An additional interface point is found in the 

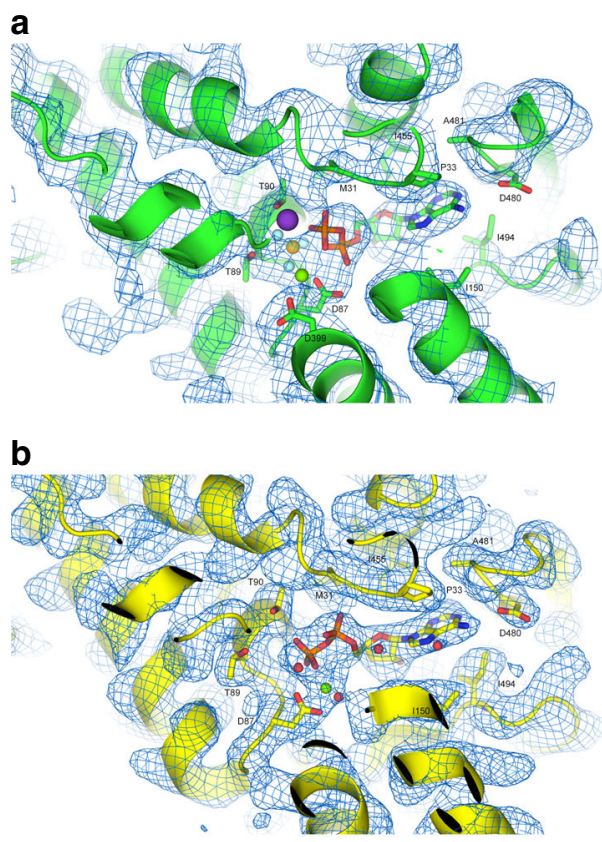

C

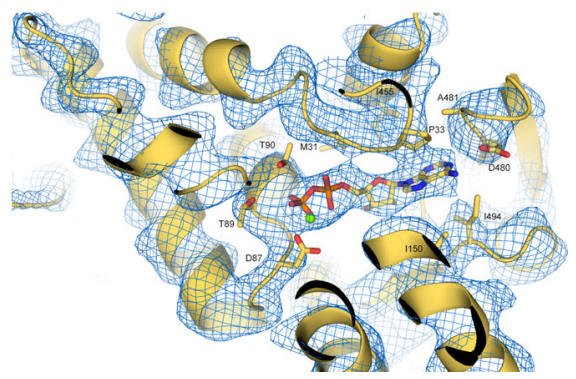

Fig. 2 Nucleotide state of the complexes. a $A D P: B F_{3}$-bound football complex. Electron-density omit map at $1 \sigma$ and the atomic model are shown around the nucleotide-binding site with one bound ADP molecule and $\mathrm{BeF}_{3}$. Selected residues in the nucleotide binding pocket are indicated. The $\mathrm{Mg}^{2+}$ ion is marked as a green sphere, $\mathrm{K}^{+}$as purple, and $\mathrm{BeF}_{3}$ as gold surrounded by cyan spheres. $\mathbf{b}$ ADP-bound football complex. Details of the cryo-EM density and the atomic model around the nucleotide-binding site with one bound ADP molecule are shown. Selected residues in the nucleotide binding pocket are indicated. The $\mathrm{Mg}^{2+}$ ion is marked as a green sphere, and water molecules are represented as red spheres. c ADP-bound halffootball complex. Details of the cryo-EM density and the atomic model around the nucleotide-binding site with one bound ADP molecule. Selected residues in the nucleotide-binding pocket are indicated. $\mathrm{The}^{\mathrm{Mg}^{2+}}$ ion is marked as a green sphere.

apical domains; namely residues of helix $\mathrm{K}$ from one subunit interact with a loop between helix G2 and strand seven of its neighbor. Helices $\mathrm{K}$ and L form a helical hairpin, a so-called K/L protrusion $^{18,33}$ that makes up a substantial portion of the wall of the folding cavity. The positioning of the interaction varies from interface to interface along a face of helix $\mathrm{K}$ in the ground-state complex, a source of subunit conformational asymmetry, while it is fixed in the ADP football (Supplementary Fig. 8b, c) in a manner that minimizes the window of the mHsp60 folding cavity.

Finally, the C-terminal 22 amino acid-long tail ending with two GGM repeats has been proposed in GroEL to protrude from the equatorial domains into each ring cavity, thereby occluding free passage between cavities ${ }^{34}$. While density for the complete GGM tail is not visible in our structures, in line with the fact that this region is predicted to be unstructured, we did observe extended density radiating into the center of the ring for the ADP football.
We modeled several residues not visible for the ground state structure, suggesting a graded ordering of this barrier between cavities as the reaction cycle progresses.

Transition from subunit conformational asymmetry to symmetry. Structural studies of GroEL have shown that its subunits undergo large conformational changes driven by ATP binding and hydrolysis along the reaction cycle (reviewed in ref. ${ }^{20}$ ). These conformational changes have been proposed to progress via structural intermediates arising from the concerted movement of all seven apical domains within one ring 22,35 . This concerted movement leads to conformational symmetry among identical subunits. Notably, the ADP: $\mathrm{BeF}_{3}$ football structure presented here displays subunit conformational asymmetry, as determined from a superposition analysis of all the mHsp60 subunits (Supplementary Table 3) and consistent with our inability to perform non-crystallographic averaging (see "Methods"). Subunit conformational variability in the $\mathrm{ADP}: \mathrm{BeF}_{3}$ football ranges from 0.3 to $2.2 \AA$ RMSD (Fig. 3a), and largely localizes to the intermediate and apical domains. In addition, we performed an alternate conformational analysis of the football structures using a cylindrical coordinate system previously employed to analyze the conformation of individual subunits in GroEL ${ }^{36,37}$. In this analysis, $\mathrm{R}$ is the distance between the $\mathrm{C} a$ of a given residue and the sevenfold symmetry axis, while $\mathrm{H}$ is defined as the height of the $\mathrm{Ca}$ of a given residue over the twofold plane of symmetry between two rings. As seen in Fig. 3b, we can discern significant asymmetry in the intermediate and apical domains for the ADP:BeF football structure. The observed subunit conformational asymmetry does not appear to be caused solely by crystal packing, since subunits not involved in crystal contacts nonetheless vary from each other.

We examined this same question for the ADP football structure determined by cryo-EM. To this end, we built a refined structure model from a $3 \mathrm{D}$ reconstruction at $3.74 \AA$ resolution determined without imposing any symmetry (C1 symmetry) (Supplementary Fig. 9). The conformational analysis described above (Fig. 3b) showed the unsymmetrized cryo-EM structure displays perfect symmetry, indicating that in solution the ADPbound football does not display subunit conformational asymmetry. Together, our analysis shows a transition from the ADP: $\mathrm{BeF}_{3}$-bound football complex, displaying significant subunit conformational asymmetry, to a conformationally symmetric ADP-bound football complex. This transition along the reaction cycle might be a unique feature of the human mitochondrial chaperonin.

A dramatic structural change in the transition from the ADP: $\mathrm{BeF}_{3}$-bound ground-state mimic to the ADP-bound football occurs with a rotation of the north pole mHsp10 lid between seven and fifteen degrees (Fig. 3c). This rotation or twisting is not uniform since the ground-state football itself is conformationally asymmetric, and is observed when using the north equatorial domain as the template for superposition. Strikingly, the south pole mHsp10 lid aligns almost perfectly (Fig. 3c). This twist is enabled by the plasticity of the apical domains in their interface with the mHsp10 mobile loops.

Next, we sought insight into the dynamic properties of the assemblies, since such information is likely to point to mobile or flexible structural components in the cycle. When we mapped the individual isotropic B-factors on the ground-state football (Fig. 3d), we discovered that the equatorial domain in $\mathrm{mHsp60}$ is most rigid, while the apical domain is most flexible, along with $\mathrm{mHsp} 10$. For the ADP:BeF ${ }_{3}$ football, there is a rotationally graded B-factor asymmetry in apical domains around the sevenfold axis, with different polarity for each pole. In addition, the mHsp10 
a

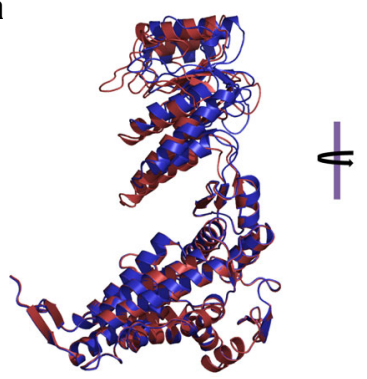

C

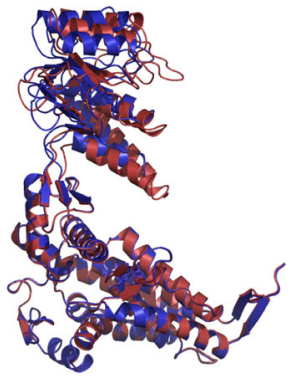

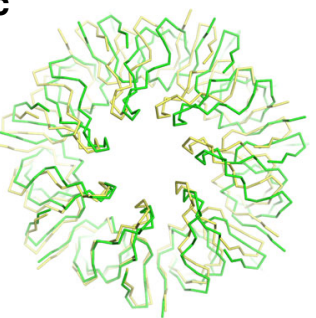

North pole

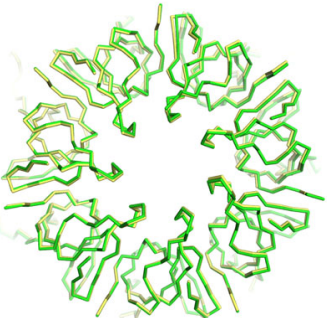

South pole

b
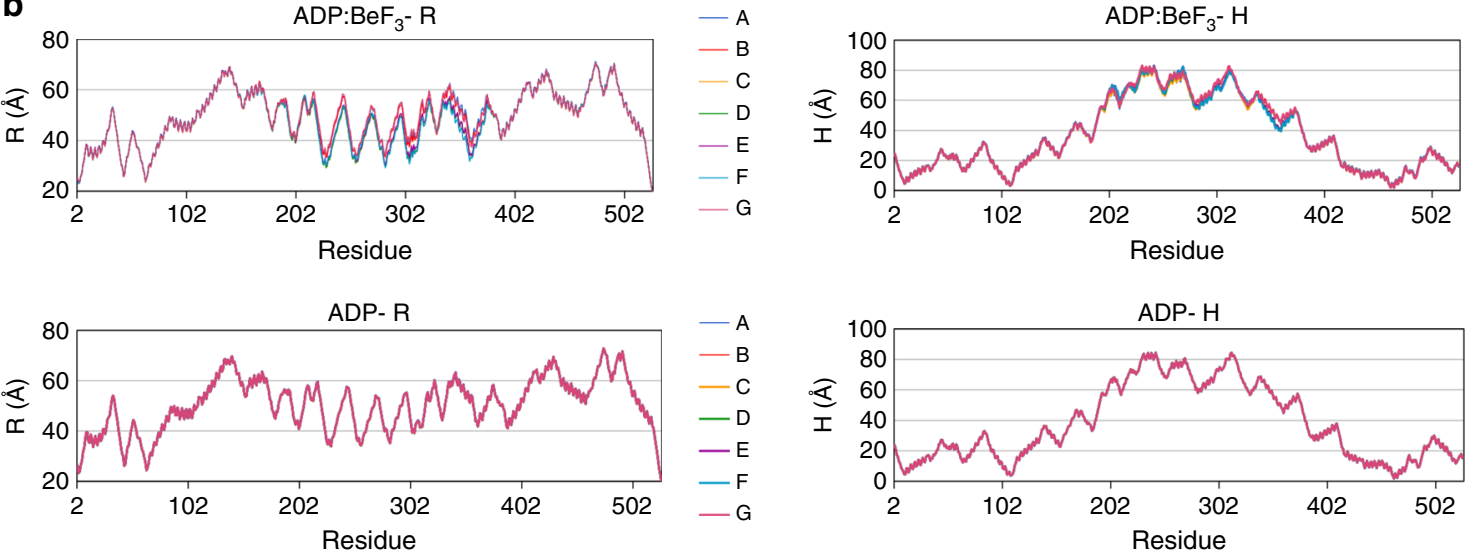

d
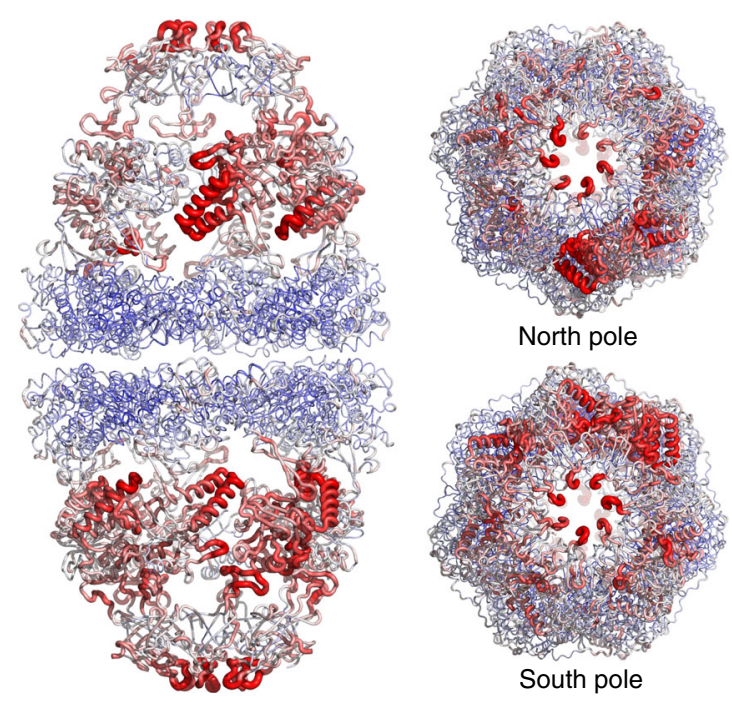

Fig. 3 Transition from the ATP ground-state mimic to ADP football. a Superposition of the two most conformationally divergent mHsp60 subunits from the $\mathrm{ADP}: \mathrm{BeF}_{3}$-bound football complex shows significant backbone conformational asymmetry. The two perspectives depicted are related by $180^{\circ}$ rotation. b The crystal structure of the ADP:BeF 3 -bound football complex and the structure of the ADP-bound football complex built from the C1 cryo-EM map were subjected to a conformational analysis using a cylindrical coordinate system previously employed to analyze the conformation of individual subunits in GroEL ${ }^{36,37}$. In this analysis, $\mathrm{R}$ is the distance between the $\mathrm{C} \alpha$ of a given residue and the sevenfold symmetry axis, whereas $\mathrm{H}$ is defined as the height of the $\mathrm{C} \alpha$ of a given residue over the twofold plane of symmetry between two rings. Each trace represents one mHsp60 subunit within the same ring. From top to bottom: $A D P: B F_{3}-R$ : radial distance to the longitudinal axis for the ADP:BeF 3 -bound football complex crystal structure; ADP-R: radial distance for the

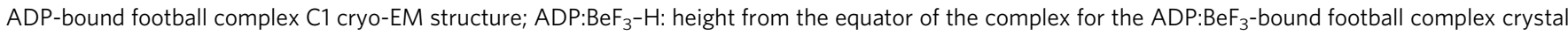
structure; ADP-H: height from the equator for the ADP-bound football complex $\mathrm{C} 1$ cryo-EM structure. c Top views of superposed $A D P: B e F_{3}$ and $A D P$ footballs in green and yellow, respectively. The north equatorial domains of $\mathrm{mHsp} 60$ were the templates for superposition. The rotation of the $\mathrm{mHsp} 10$ lid is clearly seen in the north pole, while in the south pole the structures coincide with little deviation. $\mathbf{d}$ B-factor analysis of the ground-state ADP:BeF3 crystal structure football complex. B factors are depicted by color and thickness of the worm representation. Blue and thin indicates low values while red and thick indicates high values, respectively. Side, south, and north pole views are shown. 
$\beta$-barrel mobility is diminished compared to $\mathrm{mHsp60}$ apical domain sections. We conclude that the subunit conformational asymmetry observed for the ground state football may represent snapshots of different dynamic subunit conformations, which are consistent with the B-factor analysis and provide a basis for an intra-ring communication mechanism akin to that observed for $\mathrm{CCT}^{38}$.

Unique inter-ring interface rearranges in the reaction cycle. The structures of the ADP footballs and half-footballs provide potential evidence for a second level of structural dynamics along the reaction cycle, namely the inter-ring split of mHsp60mHsp10 football complexes. To understand the structural basis for this possible pathway, we performed a comparison of several structural parameters between the structures of the three reaction cycle intermediates. The differences between the ADP football and half-football are quite small (RMSD $\sim 0.5 \AA$ ), and indicate that these structures are essentially the same, saving for the quaternary structure difference (football vs. half-football).

In contrast, when comparing the $\mathrm{ADP}: \mathrm{BeF}_{3}$ football to $\mathrm{ADP}$ football, the RMSD values range from 0.7 to $1.8 \AA$, respectively (Supplementary Table 4), mainly due to significant conformational changes in the apical and intermediate domains of the mHsp60 subunits. The volume of the ground-state football is $2.5 \%$ larger than the ADP football, where it is also $3 \AA$ longer along its sevenfold axis. The buried surface area for all the individual halves $\left(\mathrm{mHsp}_{7}-\mathrm{mHsp}_{7} 0_{7}\right)$ are about the same $\sim 6400 \AA^{2}$, except for the south half of the ground-state football, again emphasizing this assembly's subunit asymmetry (Supplementary Table 5). Notably, the inter-ring interface buried surface area for the $\mathrm{ADP}: \mathrm{BeF}_{3}$ ground state mimic is markedly larger $\left(>1000 \AA^{2}\right)$ than for the ADP football. This differential points to perhaps the most critical divergence of the two football structures, namely the geometry and interactions of the inter-ring interface.

In the ground-state football (Fig. 4a), two loci of interactions stitch the rings together, dubbed right and left (perspective from inside the assembly). The right locus involves a twofold symmetric interaction between S464 residues of apposite rings, buttressed by a H-bond from E462. This H-bond interaction has not been observed in GroEL, which relies at the right locus on an inter-ring salt bridge between the residues E461 and R452 ${ }^{18}$. The left locus involves a salt bridge from residues located on the loop between helices D and E, K109 and E105 of interfacing subunits. The corresponding inter-ring residues in GroEL are K105 and A109 that interact by virtue of the helix dipole of helix $\mathrm{D}^{39}$. We note that helix D's N-terminus forms the nucleotide-binding pocket (schematically depicted in Fig. $4 \mathrm{~b}$ ) and the loop preceding it binds the nucleotide $\gamma$ phosphate (or its equivalent here, $\mathrm{BeF}_{3}$ ). Moreover, in the $\mathrm{ADP}: \mathrm{BeF}_{3}$ football the equatorial gap between the two rings is $3.5 \AA$, as measured from planes delimited by the $\mathrm{Ca}$ of mHsp60 residue 465. In contrast, the ADP football has lost the left interaction locus, and the double mHsp60 rings are held together solely by the S464 interaction. Concomitantly, the equatorial gap has increased to $4.6 \AA$ with the buried surface area diminished, consistent with a football poised to split into its two halves. Further, when we superimposed the ADP:BeF 3 and $\mathrm{ADP}$ football structures (Fig. 4c), we discern a subtle but distinct concerted pivot of the ADP equatorial domains away from the interface plane, abrogating the left locus and giving rise to the single point S464 interaction. Biochemical and structural evidence has shown that communication between two GroEL rings is crucial for GroES release ${ }^{40}$, and that the ionic interactions at both left and right contact sites are involved in the transmission of the allosteric signal ${ }^{41,42}$. Our structural evidence for mHsp60mHsp10, however, points to minimal contacts holding the two
mHsp60 rings together (Fig. $4 \mathrm{~b}$ ), in a manner consistent with the lack of inter-ring negative cooperativity observed in biochemical experiments $^{21}$.

Given these structural findings, we tested whether the S464 residue is a critical interactor mediating the assembly of mHsp60 double rings. A bioinformatics analysis of $120 \mathrm{mHsp} 60$ orthologue sequences showed the amino acid serine to be conserved at this position, followed by alanine (Fig. 5a). Previous studies demonstrated that when ATP was readily present at all steps during electrophoresis ${ }^{28}$, or in the presence of a chemical cross-linker ${ }^{32}$, the shift to double rings in $\mathrm{mHsp} 60$ can be detected by native gels. Under conditions where ATP or its analogues are present only during pre-incubation, but omitted from all subsequent electrophoresis steps, the S464C mHsp60 mutation prevented inter-ring separation (Fig. 5b). This stabilization was due to disulfide formation during the pre-incubation, as inferred from the absence of double-ring stabilization in the presence of DTT or for the S464A variant (Fig. 5c, d). Next, we examined the ability of S464A and S464C variants to assist the refolding of model substrates (Fig. 5e). Both variants were able to assist the folding of citrate synthase (CS) to a level comparable to that displayed by WT mHsp60, but were impaired in their ability to assist the folding of malate dehydrogenase ( $\mathrm{MDH})$. Notably, the S464C variant was also impaired in its ability to assist the folding of a circular permutant (D116/G117) of enhanced green fluorescent protein (D116/G117-eGFP), shown to strictly depend on GroEL for its in vitro refolding ${ }^{43}$. These data suggest that substitution of amino acid residue S464 may affect negatively the ability of mHsp60 to assist the refolding of certain substrates.

Functional single and double mHsp60 ring complexes. As mentioned above, while active GroEL-GroES is invariably found in the double-heptameric ring form ${ }^{19,20}$, mHsp60 can be found as single- and double-ring species ${ }^{21}$. Our SEC-MALS analysis (Supplementary Fig. 7) and the cryo-EM structures of the ADP football and half-football (Fig. 1b, c) complexes are consistent with these findings. Here we addressed the question of whether single- and double-ring complexes could function independently from each other, or if alternation between them was necessary for mHsp60-mHsp10 folding activity. To this end, we generated inter-ring S464R and S464R/K109E mHsp60 variants, which based on our football structures we predicted would prevent double ring formation. SEC-MALS analysis confirmed that in the presence of ATP and $\mathrm{BeF}_{3}$, under conditions where WT mHsp60 and the S464C variant formed stable football complexes with mHsp10, both S464R and S464R/K109E mHsp60 variants assembled into single rings without evidence of higher oligomeric species (Fig. 6a). Next, we examined the ability of S464R and S464R/K109E mHsp60 single-ring variants to assist the refolding of MDH and D116/G117-eGFP as a function of ATP concentration (Fig. 6b, c). The S464R single-ring variant was significantly impaired in its ability to refold $\mathrm{MDH}$ and D116/G117eGFP, while the S464R/K109E single-ring variant was impaired in its ability to refold only D116/G117-eGFP. Thus, although reduced compared with $\mathrm{WT} \mathrm{mHsp} 60$, both these obligate singlering variants displayed significant activity against these substrates. Notably, the mHsp60 S464C cysteine-trap variant, shown above to be an obligate double-ring complex in the absence of DTT, displayed a significantly higher rate of refolding of D116/G117eGFP compared with WT mHsp60 and the obligate single-ring S464R and S644R/K109E variants (Fig. 6d). Taken together these data confirm that half-football and football complexes can assist in vitro folding independently of each other, while the double ring complexes can be more active. 

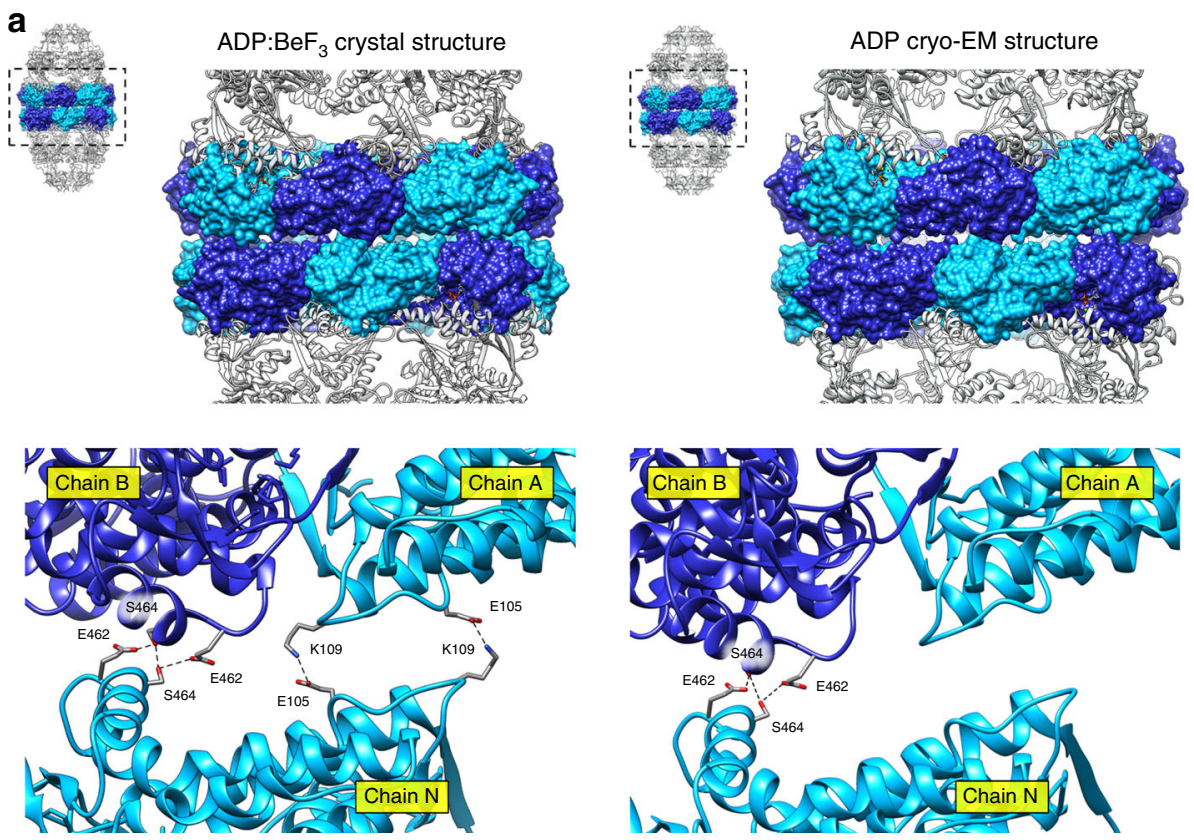

b
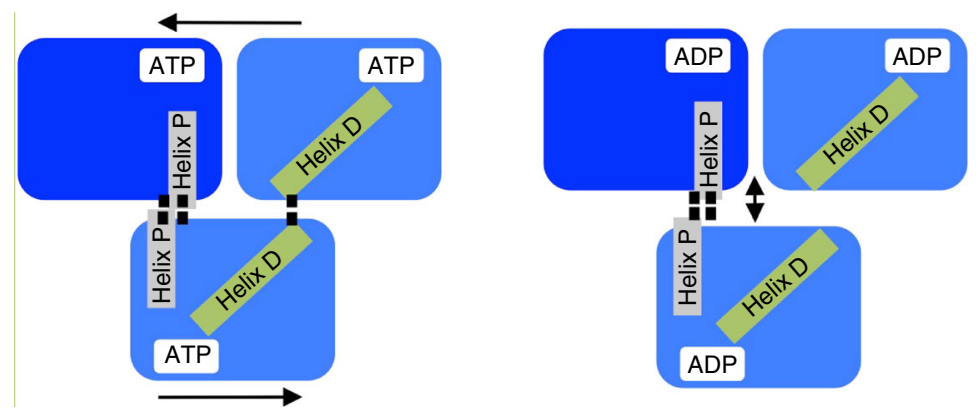

C

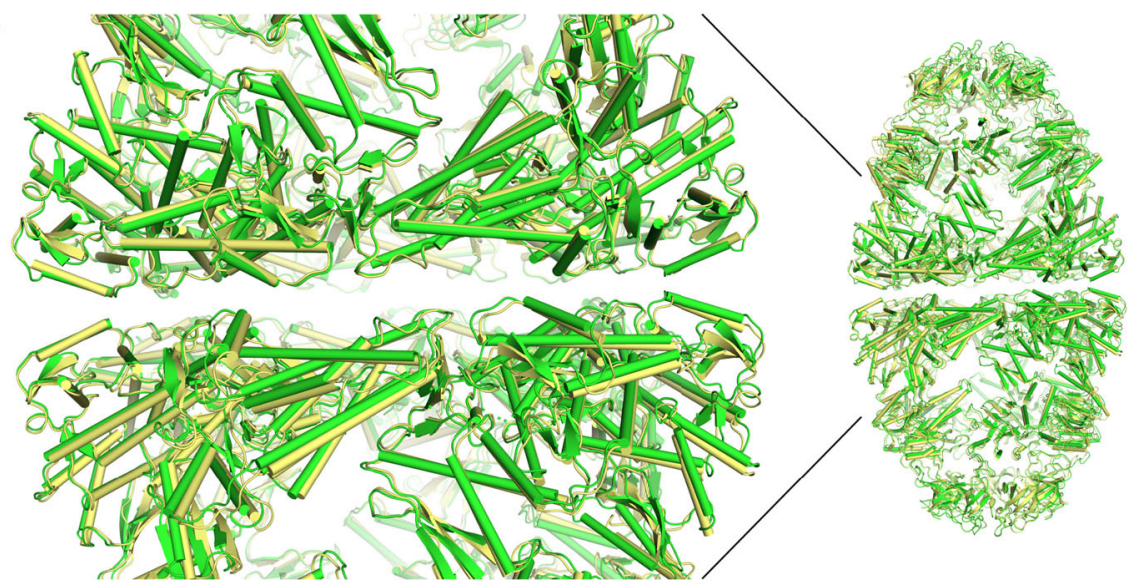

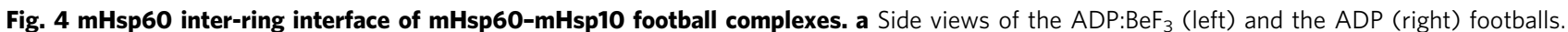
The equatorial domains of the $\mathrm{mHsp} 60$ subunits are rendered as a molecular surface in alternating blue and light blue. The other domains in the $\mathrm{mHsp60}$ subunits and $\mathrm{mHsp10}$ subunits are presented as ribbon diagrams. Below is a close-up view of the staggered arrangement of two $\mathrm{mHsp60}$ subunits in one ring contacting one subunit in the opposite ring represented as ribbon diagrams. Residues engaged in inter-ring contacts are presented as sticks. In the ADP:BeF 3 football, these are E105, K109, E462, and S464 (left). In the ADP football, these are S464 and E462 (right). Black dashed lines denote bonds between the interacting residues. $\mathbf{b}$ Cartoon representing the differences in the interactions between left (Helix D) and right

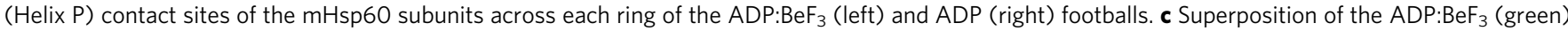
and ADP (yellow) football structures showing a close-up of the $\mathrm{mHsp} 60$ inter-ring region. 
a

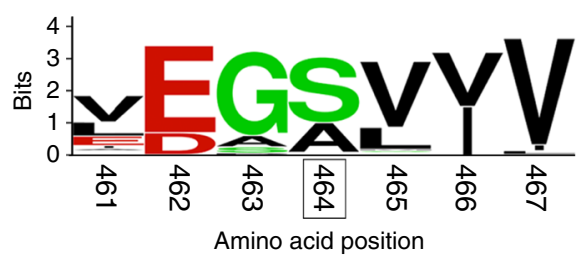

C

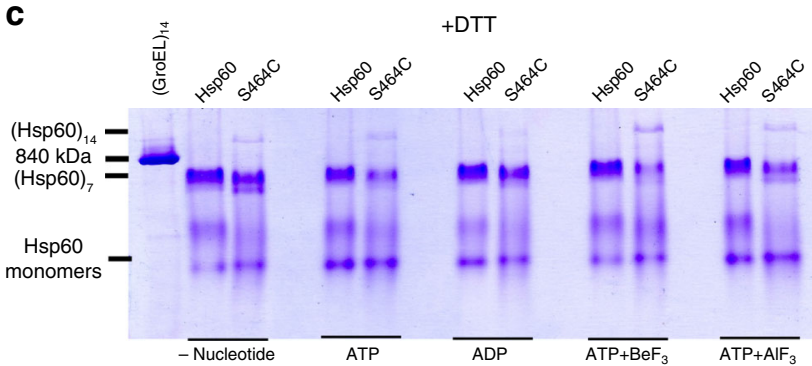

e b

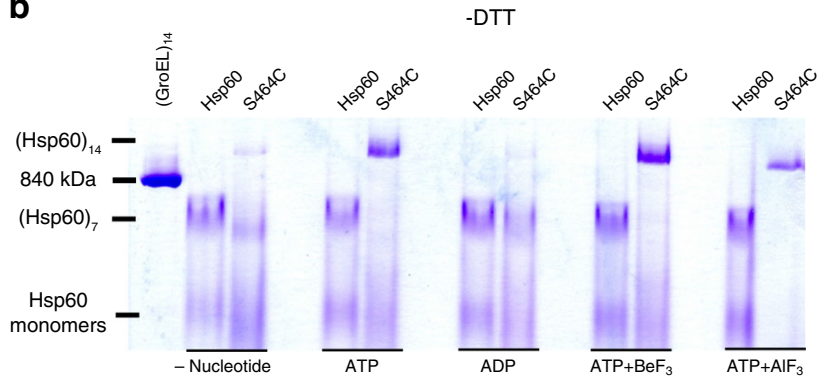

d

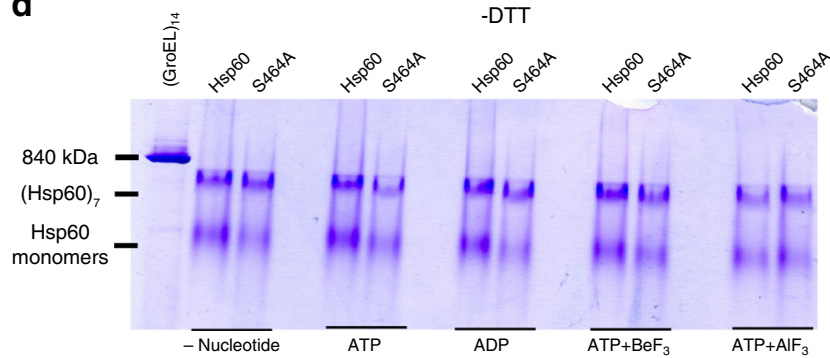

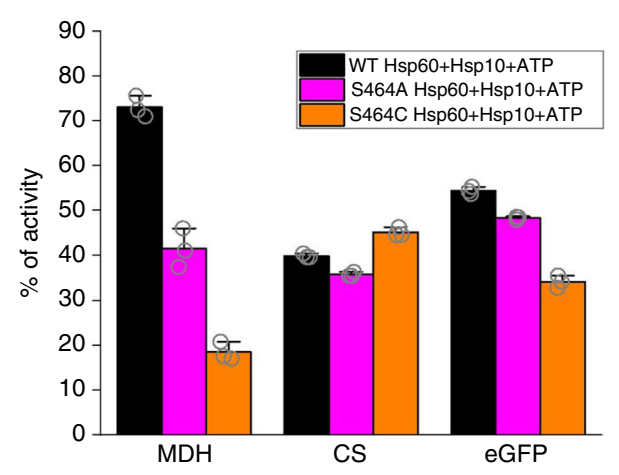

Fig. 5 Residue S464 plays a pivotal role in the assembly of $\mathbf{m H s p 6 0}$ double rings. a Logo depiction of amino acid frequency of the respective residue positions in $120 \mathrm{mHsp60}$ orthologue sequences. Amino acids are colored according to their chemical properties: polar amino acids are green, basic blue, acidic red, and hydrophobic amino acids are black. b, c Native gel analysis of WT mHsp60 and the S464C variant assembly behavior, under oxidizing (labeled - DTT) and reducing (labeled + DTT) conditions. In the experiments with nucleotide, it was added only during the pre-incubation with the chaperonin, but omitted from the loading buffer, gel, and running buffer, and thus nucleotide is effectively depleted during the electrophoresis due to separation from the protein pool and hydrolysis. d Native gel analysis of WT mHsp60 and S464A variant protein assembly behavior, under oxidizing (-DTT) conditions. The gels are representative of at least two independent experiments. e Refolding of substrate proteins MDH, CS, and D116/G117-eGFP by WT mHsp60 and its listed variants. Data represent average \pm s.d. from three independent experiments $(n=3)$. Source data are provided as a Source Data file.

We next examined whether the single-ring mutants are able to complement the GroEL-GroES deleted E. coli MGM100 strain, with the caveat that the native environment of mHsp60 is the human mitochondria and not bacteria. Both these single-ring mutants were as competent as WT mHsp60 in sustaining cell growth at 30 and $37^{\circ} \mathrm{C}$ (Fig. 6e, f), indicating that half-footballs can also function in vivo independently of footballs. Overall, we conclude that double-ring complexes might provide advantage in vitro under limiting conditions for certain substrates, however, there appears to be no advantage for WT mHsp60 compared with the single-ring mutants in E. coli.

\section{Discussion}

This work represents a major advance toward unraveling the structural basis underlying the function of group I chaperonins,

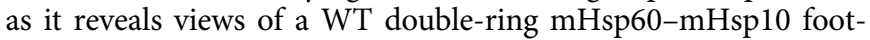
ball complex mimicking the ATP-bound ground-state intermediate and the changes in symmetry and at the inter-ring interface resulting from ATP hydrolysis. We also provided structural details of half-football complexes, and experimental evidence suggesting that active single- and double-ring mHsp60 complexes coexist in the reaction cycle of the human mHsp60-mHsp10 chaperonin system.

Specifically, our work provides a structural basis for three

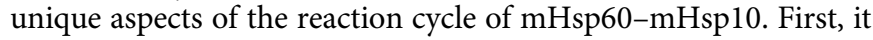
was known that nucleotides regulate the oligomeric state of mHsp60 tetradecamers ${ }^{44}$. In contrast to GroEL, ATP in mHsp60 serves as a molecular linchpin for heptamer assembly and subsequent association of heptameric rings. Based on the structural changes observed along the reaction cycle and the effect of nucleotide on the oligomeric state of the variant S464C, we propose that in the context of the steady-state (i.e., $1 \mathrm{mM}$ ATP concentration as found in the cell) ATP modulates oligomerization constants thus shifting the equilibrium toward the doublering state. Indeed, the nucleotide's primary function may be inducement of oligomerization, as opposed to providing energy for apical and intermediate domains' conformational shift, as observed in GroEL ${ }^{45}$.

Second, the labile nature of mHsp60 oligomers, in particular between rings, points to a system that employs a mechanism devoid of ATP-binding negative inter-ring cooperativity. 
a
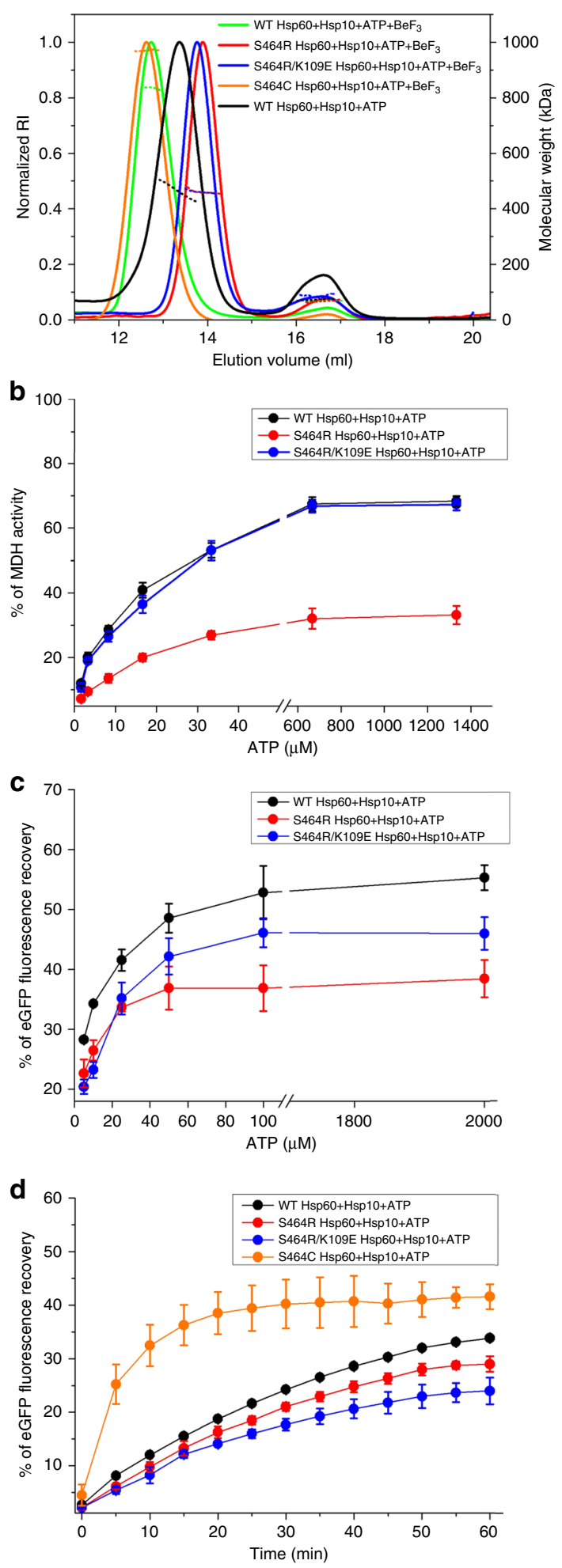

Indication for such a possibility was initially obtained from analyzing the ATPase activity of the system ${ }^{21,26,29}$. In the case of GroEL, negative cooperativity is supported structurally by the fact that under native conditions, in all structures reported, the same nucleotide type never occupies the two rings. The two exceptions are crystal structures of ground-state football GroEL obtained in the presence of $\mathrm{BeF}_{3}{ }^{37}$ or using a variant defective in ATP hydrolysis $^{46}$. For cryo-EM, we mixed mHsp60 and mHsp10 in e

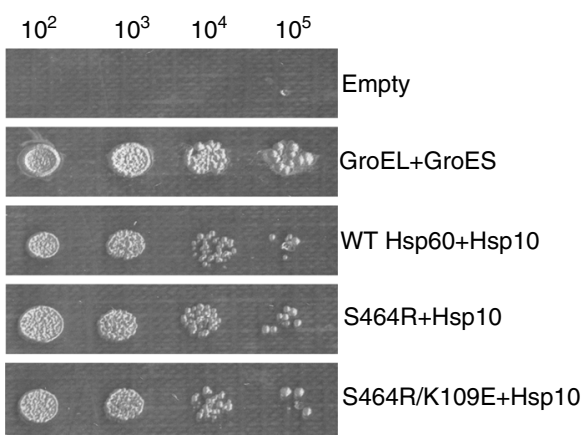

f

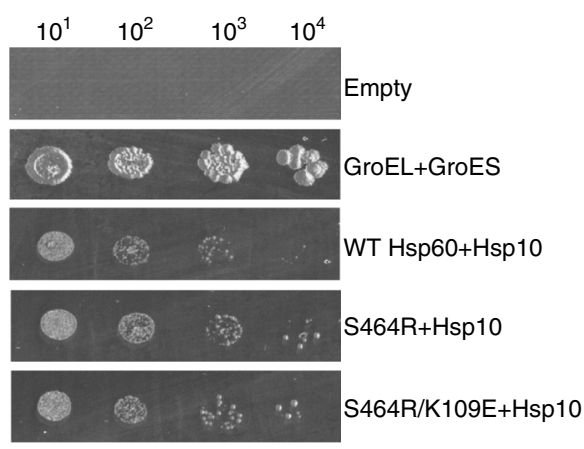

9

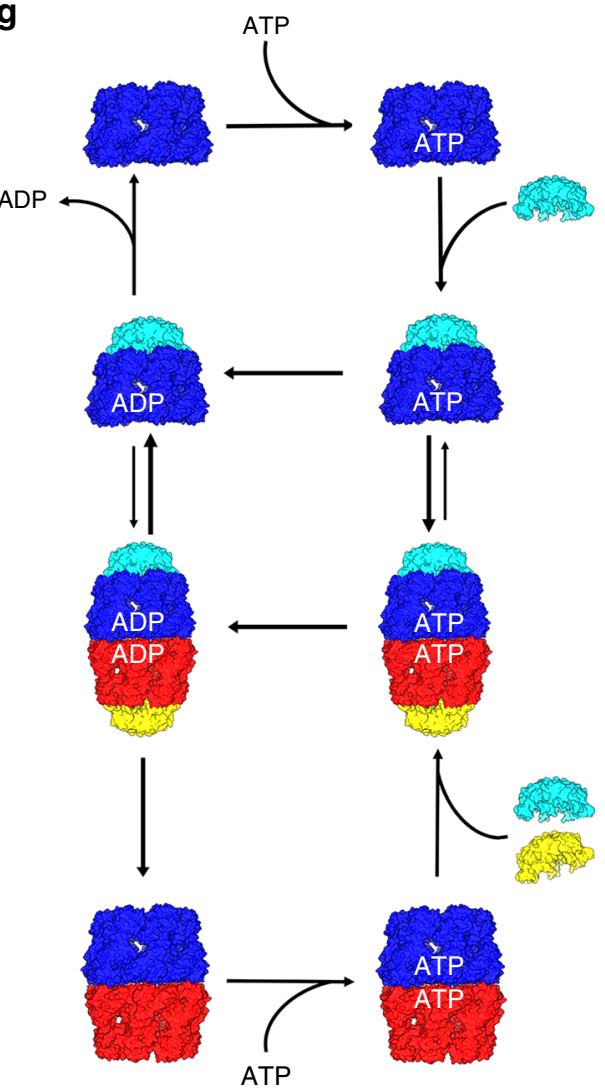

the presence of ATP, followed immediately by adsorption onto a grid and vitrification. However, all the nucleotide-binding sites in our football and half-football WT mHsp60-mHsp10 structures were occupied by ADP, indicating that under our specimen preparation conditions ATP hydrolysis had occurred rapidly. The homogeneous distribution of nucleotides among subunits provides direct evidence for the absence of negative cooperativity between rings of $\mathrm{mHsp} 60$, and indicates the ability of the rings to 
Fig. 6 Obligate single $\mathbf{m H s p 6 0}$ rings assist folding in vitro and in vivo. a SEC-MALS profiles of WT mHsp60-mHsp10 (green trace, average MWs of first and second peaks are 823 and $68 \mathrm{kDa}$, respectively), S464C mHsp60-mHsp10 (orange trace, average MW of first peak is $970 \mathrm{kDa}$ ), S464R

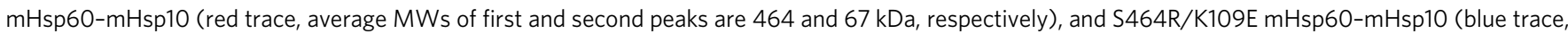
average MWs of first and second peaks are $464 \mathrm{kDa}$ and $77 \mathrm{kDa}$, respectively). The proteins were pre-incubated with $1 \mathrm{mM} \mathrm{ATP}, 1 \mathrm{mM} \mathrm{BeCl} 2$, and $10 \mathrm{mM}$ $\mathrm{NaF}$ for $5 \mathrm{~min}$. The profile of WT mHsp60-mHsp10 pre-incubated with $1 \mathrm{mM}$ ATP for $5 \mathrm{~min}$ is shown in black trace (average MWs of first and second peaks are 462 and $73 \mathrm{kDa}$, respectively). The SEC profiles are plotted against the normalized refractive index (RI) and the expected molecular mass (dashed lines). b, c Refolding of MDH and D116/G117-eGFP substrates by WT and single-ring mHsp60 variants in the presence of ATP and mHsp10. All the refolding experiments were performed at $37^{\circ} \mathrm{C}$, and data represent average \pm s.d. d Refolding of D116/G117-eGFP under oxidizing conditions by WT $\mathrm{mHsp60}$ and its listed variants. Data represent average \pm s.d. from three independent experiments $(n=3)$. e, $\mathbf{f}$ Tenfold serial dilutions of GroEL-GroESdeficient $E$. coli strain MGM100 transformed with plasmid pOFX bearing the indicated $\mathrm{mHsp} 60$ mutants and WT mHsp10. Colonies were grown on agar plates in the presence of glucose and IPTG at $30^{\circ} \mathrm{C}(\mathbf{d})$ and $37^{\circ} \mathrm{C}(\mathbf{e}) \cdot \mathbf{g}$ Revised model for the reaction cycle of mHsp60-mHsp10 based on the findings of this study. Color coding is the same as in Fig. 1a. Source data for panels a-d are provided as a Source Data file.

act as independent engines. Despite the homogenous nucleotide identity, the conformation of the subunits within and between rings of the ground state is different and exhibits conformational asymmetry. Subsequently, in the transition from the ground state (ATP football) to the ADP football, all subunits shift to both nucleotide and conformationally identical states. We argue that this transition to subunit symmetry might be related to the accepted notion in the field of protein oligomerization, that a symmetric assembly is at a lower energy state than its asymmetric counterpart ${ }^{47,48}$. In the context of mHsp60, ATP hydrolysis will be associated with asymmetry, but subsequently the system relaxes into the symmetric and lower energy ADP-bound state.

Third, previous in vitro analysis suggested that mHsp60 undergoes inter-ring split (also termed equatorial split) during its reaction cycle 21,26 . However, at which stage in the reaction cycle of mHsp60 would the rings separate was unknown. Recently, a possible equatorial split has also been reported for GroEL ${ }^{49}$, although the necessity of ring splitting for the function of GroEL is still controversial, as linking of both rings by either formation of S-S bonds ${ }^{49}$ or covalent fusion still allows for significant protein-folding activity ${ }^{50}$. From our cryo-EM images showing abundant ADP-bound football and half-football complexes, we postulated the former might represent a pre-split intermediate. Native gel analysis of the cysteine-trap variant (S464C) provided direct evidence for the modulation of single ring to double ring structures by nucleotides. Clearly, in the absence of nucleotides or the presence of $\mathrm{ADP}$, the mHsp60 molecules assemble into single-ring structures, while in the presence of ATP or its nonhydrolysable analogues it forms double-ring structures.

In order to test whether the validity of our hypothesis that the functional cycle proceeds via an equatorial split comprised of alternating single-ring and double-ring assemblies, we generated two types of variants. One type consists of obligate single-ring mHsp60 (S464R and S464R/K109E variants), while the other consists of obligate double rings (S464C cysteine-trap variant). Experiments with the single-ring variants demonstrate that while under certain conditions they are less efficient in assisting substrate-protein folding, they are nonetheless functional without the requirement to form double rings.

Obligate mHsp60 double-ring assemblies were trapped by formation of inter-ring disulfide bonds in the absence of reducing agent (Fig. 5b). If inter-ring split is essential then the refolding activity of the complex ought to be compromised in the absence of reducing agent, but not in its presence. Strikingly, the cysteinetrap variant was even more active than WT and the two singlering variants in the absence of reducing agent. Hence, our results indicate that both single and double rings are functional and that the equatorial split is not essential for the folding activity of the mHsp60-mHsp10 chaperonin system, in vitro, or in bacteria.

In light of these findings, we have updated our initial model to include two separate folding cycles with single-ring and double- ring pathways, with the potential of crossing over upon equatorial split of the double rings (Fig. 6g). In this model, the occupancy of nucleotide-binding sites by ADP triggers the dissociation of mHsp10, consistent with reports showing that mHsp60 binds mHsp10 in the presence of ATP, AMPNP and ADP-BeF 3 , but not $\mathrm{ADP}$ and $\mathrm{ADP}-\mathrm{AlF}_{3}{ }^{21,26}$. In line with $\mathrm{mHsp60}$ nucleotidedependence studies ${ }^{21,29}$, exchange of ADP would occur in the presence of excess ATP.

These separate cycles then raise the question as to what might be the biological advantage of two possible modalities for mHsp60-mHsp10, i.e., assisted folding by way of footballs or half-footballs. We speculate that the individual cycles may be optimized for different substrate sets, thereby expanding and tuning this chaperonin system for its host of substrates in mitochondria, in an analogous manner as recently suggested for E. coli GroEL-GroES ${ }^{51}$

\section{Methods}

Protein expression and purification. mHsp60 is translated in the cytosol as a 573 amino acid-long polypeptide containing a 26 amino acid-long mitochondrial Matrix Targeting Signal (MTS), which when cleaved yields the mature 547 amino acid-long mitochondrial protein (Supplementary Fig. 1a). Human mt-cpn60 gene, lacking the N-terminal mitochondrial MTS, was cloned into a modified pET21d plasmid $^{52}$. The N-terminal HisTagged protein with an engineered TEV cleavage site was overexpressed in E. coli Rosetta ${ }^{\mathrm{TM}}$ strain for $3 \mathrm{~h}$ at $25^{\circ} \mathrm{C}$ following induction by $1 \mathrm{mM}$ IPTG at $\mathrm{OD}_{600}=0.6$. The harvested cells were suspended $(1: 10$ w/w) in buffer (100 mM Tris- $\mathrm{HCl} \mathrm{pH} 7.7,10 \mathrm{mM} \mathrm{MgSO} 4,1 \mathrm{mM} \beta-\mathrm{ME}, 5 \%$ glycerol, $0.1 \%$ Triton X-100 and protease inhibitors) and lysed using a microfluidizer. Following centrifugation for $30 \mathrm{~min}$ at $35,000 \mathrm{xg}$, the supernatant was diluted (1:3 $\mathrm{w} / \mathrm{w})$ in buffer $(10 \mathrm{mM}$ Tris- $\mathrm{HCl} \mathrm{pH} 7.7,5 \%$ glycerol, $0.1 \%$ Triton X-100, and 10 $\mathrm{mM}$ imidazole), loaded onto a nickel-agarose resin column (equilibrated with the latter buffer) and eluted with $250 \mathrm{mM}$ imidazole. Fractions containing mt-cpn60 were pooled and, upon addition of TEV protease $(1: 30 \mathrm{w} / \mathrm{w})$, dialyzed overnight against $20 \mathrm{mM}$ Tris- $\mathrm{HCl} \mathrm{pH} \mathrm{7.7,5 \%} \mathrm{glycerol,} \mathrm{and} 200 \mathrm{mM} \mathrm{NaCl}$ at $4{ }^{\circ} \mathrm{C}$. As a result of TEV cleavage and removal of the HisTag, the $\mathrm{mHsp60}$ employed for structural studies begins with the amino acid sequence GS. Next, the protein sample was diluted with sixfold volume of $20 \mathrm{mM}$ Tris- $\mathrm{HCl} \mathrm{pH} 7.7$ and 12 -fold volume of $\mathrm{H}_{2} \mathrm{O}$, loaded onto an anion-exchange column (equilibrated with $20 \mathrm{mM}$ Tris- $\mathrm{HCl} \mathrm{pH}$ $7.7,5 \%$ glycerol, $200 \mathrm{mM} \mathrm{NaCl}$, and $10 \mathrm{mM}$ imidazole), and eluted with a $100-400$ $\mathrm{mM} \mathrm{NaCl}$ gradient. Fractions containing monomeric $\mathrm{mHsp} 60$ were collected and concentrated. The concentrated sample $(18 \mathrm{mg} / \mathrm{ml})$ was then incubated at $30^{\circ} \mathrm{C}$ in the presence of $4 \mathrm{mM} \mathrm{ATP}, 20 \mathrm{mM} \mathrm{KCl}$, and $20 \mathrm{mM} \mathrm{Mg}$ acetate, in order to induce oligomerization of monomers. After $2 \mathrm{~h}$, the sample was loaded on a Superdex 200 gel-filtration column in $50 \mathrm{mM}$ Tris- $\mathrm{HCl} \mathrm{pH} \mathrm{7.7,300} \mathrm{mM} \mathrm{NaCl}$, and $10 \mathrm{mM}$ $\mathrm{MgCl}_{2}$. Fractions containing active oligomeric mHsp60 were collected, concentrated, and frozen using liquid nitrogen. GroEL and GroES were overexpressed in E. coli and purified by a combination of size-exclusion and ionic exchange chromatography 53 .

Human mt-cpn10 was expressed without a HisTag (Supplementary Fig. 1b) ${ }^{28}$ Bacterial cell pellet was resuspended $(1: 10 \mathrm{w} / \mathrm{v})$ in a buffer containing $20 \mathrm{mM}$ Tris$\mathrm{HCl} \mathrm{pH} \mathrm{7.7,} 5 \mathrm{mM} \mathrm{MgSO}_{4}, 1 \mathrm{mM}$ DTT, PMSF $(0.5 \mathrm{mM})$, and $1 \mu \mathrm{g} / \mathrm{ml}$ each of the protease inhibitors pepstatin, chymostatin, antipain, leupeptin, and aprotinin (Sigma), and 1500 units of DNase and lysed by sonication. Cellular debris was removed by centrifugation for $30 \mathrm{~min}$ at 35,000xg. The supernatant was loaded onto a RESOURCE Q column (GE Healthcare) equilibrated with buffer A ( $20 \mathrm{mM}$ Tris- $\mathrm{HCl} \mathrm{pH}$ 7.7, $0.1 \mathrm{mM}$ EDTA, and $1 \mathrm{mM}$ DTT). Eluted proteins were collected and dialyzed overnight against buffer B (20 mM MES pH 6.6 and $0.1 \mathrm{mM}$ EDTA). The protein was then loaded on a SOURCE-S column (GE Healthcare) equilibrated 
with buffer B. Bound proteins were eluted from the column with a linear gradient of 0-500 mM NaCl. mHsp10-enriched fractions were collected and concentrated. The protein was then loaded on a Superdex 200 prep grade gel-filtration column (Pharmacia) equilibrated with buffer C $(50 \mathrm{mM}$ Tris- $\mathrm{HCl} \mathrm{pH} 7.7$ and $100 \mathrm{mM}$ $\mathrm{NaCl}$ ). Afterward, purified $\mathrm{mHsp} 10$ was concentrated and flash-frozen in liquid nitrogen for storage.

For purification of D116/G117-eGFP bacterial pellets (Rosetta ${ }^{\text {tw }}$ strain (Merck)) were suspended in $50 \mathrm{mM}$ Tris buffer (pH 7.5) containing $100 \mathrm{mM} \mathrm{NaCl}, 10 \mathrm{mM}$ imidazole, and $0.1 \mathrm{mM} \mathrm{DTT}{ }^{54}$. The cells were then disrupted using sonication, and the lysate was clarified by centrifugation. The supernatant was loaded onto a $5 \mathrm{ml}$ Nickel column, and D116/G117-eGFP was eluted with $200 \mathrm{mM}$ imidazole. The eluted protein dialyzed, and afterward loaded onto Q column and eluted with gradient of 0.01-1 M NaCl. Concentrated D116/G117-eGFP was loaded onto a Superdex 75 column (Amersham Pharmacia) equilibrated with $10 \mathrm{mM}$ phosphate buffer (pH 7) containing $0.1 \mathrm{mM}$ DTT. Fractions containing D116/G117-eGFP were combined, divided into aliquots that were snap-frozen in liquid nitrogen, and stored at $-80^{\circ} \mathrm{C}$.

Crystallization. The football complex was made by mixing $215 \mu \mathrm{M} \mathrm{mHsp} 60,455$ $\mu \mathrm{M} \mathrm{mHsp} 10,1 \mathrm{mM}$ ATP, $1 \mathrm{mM} \mathrm{BeCl}_{2}$ and $10 \mathrm{mM} \mathrm{NaF}$ in Buffer F $(75 \mathrm{mM}$ Tris pH 7.7, $15 \mathrm{mM} \mathrm{MgCl}_{2}, 150 \mathrm{mM} \mathrm{KCl}$, and $300 \mathrm{mM} \mathrm{NaCl}$ ). Crystals containing $\mathrm{mHsp} 60$ and mHsp10 were initially grown in 10\% PEG 6000 and $2 \mathrm{M} \mathrm{NaCl}$ at 293 K. Optimized, diffraction-quality, crystals were grown in 10\% PEG 6000, $2.1 \mathrm{M}$ $\mathrm{KCl}$, and $\mathrm{Na}$-HEPES buffer $\mathrm{pH}$ 8. Crystals prepared for diffraction measurements were mounted in cryo-loops, frozen in the cryostream, removed, and then soaked in cryoprotectant buffer (10\% PEG 6000, $2.1 \mathrm{M} \mathrm{KCl}$, Na-HEPES buffer $\mathrm{pH} 8$ and $20 \%$ glycerol) for $5 \mathrm{~min}$ and then plunged into liquid nitrogen.

\section{Data collection and crystallographic structure determination. Diffraction data} of the mHsp60-mHsp10 ADP-BeF 3 complex crystals were collected at beamline ID29 at the European Synchrotron Radiation Facility (Grenoble, France). 1140 images with $0.1^{\circ}$ oscillation were collected at $100 \mathrm{~K}$. The data were indexed, integrated, and scaled using $\mathrm{XDS}^{55}$. The structure was solved by molecular replacement using PHASER implemented in the Phenix software suite ${ }^{56}$. The search model was a half-football mHsp60-mHsp10 ADP complex derived from the football mHsp60-mHsp10 ADP complex determined by single-particle cryo-EM, as described below, with the nucleotide removed. Subsequently, molecular replacement searches employed a heterodimeric $\mathrm{mHsp} 60-\mathrm{mHsp} 10$ structure, again derived from the cryo-EM determined mHsp60-mHsp10 ADP football complex. Both searches concluded with a football structure solution. Refinement proceeded with this model. In the first stage, rigid-body refinement was performed, dividing the $\mathrm{mHsp} 60$ protomer into three domains, equatorial, intermediate, and apical along with $\mathrm{mHsp} 10$. This round improved the electron-density maps significantly. Notably, NCS averaging degraded the maps considerably. Hence, strict NCS constraints were not used for ensuing refinement but rather NCS restraints. Rounds of standard refinement (phenix.refine) including TLS methods and manual rebuilding using $\operatorname{Coot}^{57}$ produced a model of good stereochemistry and R-factors.

Electron microscopy. Aliquots of purified $\mathrm{mHsp} 60$ and $\mathrm{mHsp} 10$ were diluted to $50 \mu \mathrm{M}$ in reaction buffer $\left(20 \mathrm{mM}\right.$ Tris- $\mathrm{HCl} \mathrm{pH} 7.7,20 \mathrm{mM} \mathrm{KCl}, 10 \mathrm{mM} \mathrm{MgCl}_{2}$, $2 \mathrm{mM}$ ATP). The complex was assembled by mixing the individual protein subunits at room temperature in reaction buffer to a final concentration of $5 \mu \mathrm{M}$ $\mathrm{mHsp} 60$ and $6 \mu \mathrm{M} \mathrm{mHsp} 10$ (molar ratio 1:1.2). The mixture was kept at room temperature at all times to avoid the formation of particle strings observed in some vitrified specimens. After only a few seconds of incubation, $3 \mu l$ of the mixture were adsorbed onto glow-discharged gold-copper homemade Lacey holey grids and immediately one-side blotted for $4 \mathrm{~s}$ from the back of the grid and flash-frozen in liquid ethane using a manual homemade cryoplunger. Overall mixing to vitrification took $\sim 30 \mathrm{~s}$. The grids were imaged on a $300 \mathrm{kV}$ Titan Krios (FEI) equipped with a K2 camera (Gatan) operating in counting mode at a calibrated pixel size of $1.07 \AA /$ pix. We recorded a single movie per hole with a maximum total accumulated exposure of $63 \mathrm{e}-/ \AA^{2}$ fractionated into 50 frames of $200 \mathrm{~ms}$ (yielding movies of $10 \mathrm{~s}$ duration). We employed a defocus range between 0.8 and $2.5 \mu \mathrm{m}$. Comafree alignment of the microscope was performed using Leginon ${ }^{58}$. In total, 1883 movies were recorded automatically with Leginon ${ }^{59}$ to control both the microscope and the K2 camera.

Image processing and EM structure determination. For data processing, frame alignment of each movie was carried out using MotionCor $2^{60}$ and calculated their contrast transfer function (CTF) values with CTFFIND4 ${ }^{61}$. The effective defocus calculated by this method ranged from 0.5 to $2.5 \mathrm{~mm}$ for the whole data set. The best 1634 movies according to CTF values and total motion were chosen for particle selection. We used FindEM ${ }^{62}$ integrated into the Appion pipeline ${ }^{63}$, to automatically select 162,669 particles using templates generated from low resolution 3D reconstructions of $\mathrm{mHsp} 60-\mathrm{mHsp} 10$ football complexes. $2 \mathrm{D}$ and $3 \mathrm{D}$ classification and refinements were performed using RELION ${ }^{64}$ without imposing symmetry. The X-ray structure of the $\mathrm{mHsp} 60^{\mathrm{E} 321 \mathrm{~K}}$ - $\mathrm{mHsp} 10$ football complex (PDB 4PJ1) filtered at $60 \AA$ was used as initial model for 3D classification. An initial refinement without imposing symmetry of a subset of 52,261 football particles generated a $4.2 \AA$ resolution 3D reconstruction (based on the goldstandard FSC $=0.143$ criterion $^{30}$ ) with a high degree of D7 symmetry. The data were then transferred to cryoSPARC ${ }^{65}$ and analyzed independently by $2 \mathrm{D}$ classification, Ab-initio modeling, and homogeneous refinement. The analysis by cryoSPARC without imposing symmetry generated an initial map at a $3.74 \AA$ resolution based on the gold-standard FSC $=0.143$ criterion $^{30}$ from 66,013 particles. Consistent with the observation of a high degree of D7 symmetry in all the reconstructions of the football particle generated both by RELION and cryoSPARC, we decided to impose this symmetry during the refinement yielding a $3 \mathrm{D}$ reconstruction of the football complex at $3.08 \AA$ resolution. A smaller subset of images of the half-football complex, consisting in 10,972 particles, generated a C7 symmetry $3.83 \AA$ map of the half-football complex. The local resolution of the $3 \mathrm{D}$ reconstructions was determined using ResMap ${ }^{66}$. For model building, we began by rigid-body fitting into our map a single $\mathrm{mHsp} 60$ (subunit $\mathrm{A}$ ) and $\mathrm{mHsp} 10$ (subunit O) subunit from the $3.15 \AA$ crystal structure of the ADP-bound mHsp60 $0^{\mathrm{E} 321 \mathrm{~K}_{-}}$mHsp10 football complex (PDB 4PJ1) ${ }^{28}$. To improve the fit and to optimize stereochemistry, we carried out real space refinement with secondary structure and geometry restraints and simulated annealing using Phenix ${ }^{67}$.

Refolding assays. Denaturation of $0.45 \mu \mathrm{M}$ pig heart MDH was performed in the presence of $5 \mathrm{mM} \mathrm{HCl}$ at room temperature. After 1-h incubation, the activity of $\mathrm{MDH}$ was measured to confirm the loss of activity. Denatured MDH was diluted into buffer (50 mM Na-HEPES pH 7.4, $50 \mathrm{mM} \mathrm{KCl,} 20 \mathrm{mM} \mathrm{MgCl}_{2}$, and $5 \mathrm{mM}$ DTT) containing mHsp60. After $30 \mathrm{~min}$ of incubation at $30^{\circ} \mathrm{C}$, in which the $\mathrm{mHsp60}$-substrate binary complex is formed, the $\mathrm{mHsp60}$-assisted $\mathrm{MDH}$ refolding was initiated by adding $\mathrm{mHsp} 10$ and ATP. The final monomer concentrations were: $10 \mu \mathrm{M}$ mHsp60, $20 \mu \mathrm{M}$ mHsp10, and $0.45 \mu \mathrm{M}$ MDH (Roche). Following $1 \mathrm{~h}$ incubation at room temperature, $20 \mu \mathrm{l}$ aliquots were taken and assayed for MDH activity by adding $980 \mu \mathrm{l}$ reaction mixture, which contained $150 \mathrm{mM}$ potassium phosphate $\mathrm{pH} 7.5,5 \mathrm{mM}$ DTT, $0.5 \mathrm{mM}$ oxaloacetate, and $0.28 \mathrm{mM} \mathrm{NADH}$. MDH activity was determined by monitoring NADH oxidation as a function of time at $340 \mathrm{~nm}$. The refolding activity is presented relative to the refolding activity of native $\mathrm{MDH}(100 \%)$

CS $(25 \mu \mathrm{M})$ was denatured at room temperature in a buffer of $6 \mathrm{M} \mathrm{Gdn}-\mathrm{HCl}$, $50 \mathrm{mM}$ Tris-HCl PH 8, $3 \mathrm{mM}$ DTT, and $2 \mathrm{mM}$ EDTA. Then, CS was promptly diluted 100 -fold into buffer $\mathrm{D}\left(0.15 \mathrm{M}\right.$ Tris- $\left.\mathrm{HCl} \mathrm{pH} 8,16 \mathrm{mM} \mathrm{MgCl}_{2}, 32 \mathrm{mM} \mathrm{KCl}\right)$ containing $30 \mu \mathrm{M} \mathrm{mHsp} 60$ at $30^{\circ} \mathrm{C}$ for $30 \mathrm{~min}$. Refolding was commenced by adding $20 \mu$ of $120 \mu \mathrm{M}$ mHsp10 and $3 \mathrm{mM}$ ATP in buffer D to $40 \mu$ of the above mixture. CS activity was assayed by using $0.5 \mathrm{mM}$ oxaloacetate and $0.2 \mathrm{mM}$ acetylcoA as substrates. The condensing reaction was monitored by binding of Ellman's reagent (DTNB) to the free SH group of the released CoA. The activity of the refolding CS is given as a percentage relative to a control sample of native CS.

D116/G117-eGFP was acid denatured in $200 \mathrm{ml}$ of $30 \mathrm{mM} \mathrm{HCl}$ at a final concentration of $12.5 \mu \mathrm{M}$ and incubated at $25^{\circ} \mathrm{C}$ for $1 \mathrm{~h}$. The denatured protein was then diluted 100 -fold in $200 \mathrm{ml}$ of $50 \mathrm{mM}$ (3-(N-morpholino)propanesulfonic acid) buffer (pH 7.0) containing $100 \mathrm{mM} \mathrm{KCl}, 10 \mathrm{mM} \mathrm{Mg}(\mathrm{CH} 3 \mathrm{COO})_{2}, 5 \mathrm{mM}$ DTT, and $0.0125 \%$ Tween 20 (refolding buffer). The refolding buffer contained

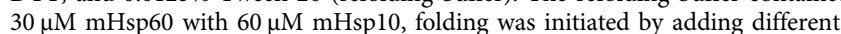
concentrations of ATP. Folding was monitored at $37^{\circ} \mathrm{C}$ using a Synergy HT plate reader (Bio Tek) with a 485/20 excitation filter, a 528/20 emission filter, and a gain of 55 . The fluorescence recovery is presented relative to the fluorescence of native D116/G117-eGFP (100\%).

Native gel analysis. WT and variant $\mathrm{mHsp} 60(15 \mu \mathrm{M})$ were incubated for $5 \mathrm{~min}$ in buffer $\mathrm{E}\left(20 \mathrm{mM}\right.$ Tris- $\left.\mathrm{HCl} \mathrm{pH} \mathrm{7.7,20} \mathrm{mM} \mathrm{MgCl}_{2}, 50 \mathrm{mM} \mathrm{KCl}\right)$ in the presence or absence of nucleotide, and with and without $1 \mathrm{mM}$ DTT. There was no additional nucleotide in the loading buffer, gel or running buffer. Hence, in this experiment nucleotide is effectively depleted during electrophoresis due to separation from the protein pool and hydrolysis, and not resupplied. We loaded 5 $\mu \mathrm{g}$ of each sample into a $6 \%$ native-PAGE.

SEC-MALS analysis. Samples were incubated at the specified solution conditions, described in the legend to Supplementary Fig. 7. After incubation for $5 \mathrm{~min}$ at room temperature $\left(\sim 25^{\circ} \mathrm{C}\right)$, the proteins were injected into a Superose- 6 SEC column (GE Healthcare) that was equilibrated with the same buffer. The column was connected to a MALS detector (DAWN HELEOS II; Wyatt Technology) and then to a refractive index detector (Optilab t-rEX; Wyatt Technology). Wyatt Astra $\mathrm{V}$ software was used for data collection and analysis. Data were collected at $\sim 25^{\circ} \mathrm{C}$.

In vivo complementation assay. Using the IPTG-inducible pOFX plasmid expressing wild-type human $\mathrm{mHsp} 10$ and $\mathrm{mHsp60}{ }^{29}$, we engineered two additional constructs containing human $\mathrm{mHsp} 10$ and the single-ring mHsp60 mutants. The mutations S464R and S464R/K109E were inserted into pOFX by Gibson assembly and transformed into E. coli NEB5a. Following purification from NEB5a, the pOFX plasmids were transformed by electroporation into MGM100 competent cells. Finally, the ability of cells were grown on 2YT-agar plates containing $25 \mathrm{mg} / \mathrm{ml}$ kanamycin and $50 \mathrm{mg} / \mathrm{ml}$ spectinomycin in the presence of either: $0.2 \%$ arabinose, $0.5 \%$ glucose or $0.5 \%$ glucose, and $1 \mathrm{mM}$ IPTG. 
Reporting summary. Further information on research design is available in the Nature Research Reporting Summary linked to this article.

\section{Data availability}

The EM maps of ADP-bound mHsp60-mHsp10 football and half-football complexes have been deposited in the Electron Microscopy Data Bank (http://www.ebi.ac.uk/pdbe/ emdb/) under accession numbers EMD-9195 and EMD-9196, respectively. The atomic coordinates of ADP-bound mHsp60-mHsp10 football, $\mathrm{ADP}: \mathrm{BeF}_{3}$ bound

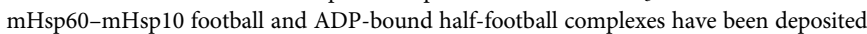
in the Protein Data Bank (www.pdb.org) under PDB 6MRC, PDB 6HT7, and PDB $6 \mathrm{MRD}$, respectively. The source data underlying Figs. 5e, 6a-d and Supplementary Figs $7 \mathrm{a}-\mathrm{c}$ are provided as a Source Data file. All reagents and relevant data are available from the corresponding authors upon reasonable request.

Received: 22 October 2018; Accepted: 17 March 2020;

Published online: 21 April 2020

\section{References}

1. Levy-Rimler, G., Bell, R. E., Ben-Tal, N. \& Azem, A. Type I chaperonins: not all are created equal. FEBS Lett. 529, 1-5 (2002).

2. Cheng, M. Y. et al. Mitochondrial heat-shock protein hsp60 is essential for assembly of proteins imported into yeast mitochondria. Nature 337, 620-625 (1989).

3. Christensen, J. H. et al. Inactivation of the hereditary spastic paraplegiaassociated Hspd1 gene encoding the Hsp60 chaperone results in early embryonic lethality in mice. Cell Stress Chaperones 15, 851-863 (2010).

4. Cappello, F., Conway de Macario, E., Marasa, L., Zummo, G. \& Macario, A. J. Hsp60 expression, new locations, functions and perspectives for cancer diagnosis and therapy. Cancer Biol. Ther. 7, 801-809 (2008).

5. Osterloh, A. et al. Lipopolysaccharide-free heat shock protein 60 activates T cells. J. Biol. Chem. 279, 47906-47911 (2004).

6. Johnson, B. J. et al. Heat shock protein 10 inhibits lipopolysaccharide-induced inflammatory mediator production. J. Biol. Chem. 280, 4037-4047 (2005)

7. Xanthoudakis, S. et al. Hsp60 accelerates the maturation of pro-caspase- 3 by upstream activator proteases during apoptosis. EMBO J. 18, 2049-2056 (1999).

8. Knowlton, A. A. \& Gupta, S. HSP60, Bax, and cardiac apoptosis. Cardiovas. Tox 3, 263-268 (2003).

9. Ban, H. S., Shimizu, K., Minegishi, H. \& Nakamura, H. Identification of HSP60 as a primary target of o-carboranylphenoxyacetanilide, an HIF-1alpha inhibitor. J. Am. Chem. Soc. 132, 11870-11871 (2010).

10. Chun, J. N. et al. Cytosolic Hsp60 is involved in the NF-kappaB-dependent survival of cancer cells via IKK regulation. PLoS ONE 5, e9422 (2010).

11. Hansen, J. et al. A novel mutation in the HSPD1 gene in a patient with hereditary spastic paraplegia. J. Neurol. 254, 897-900 (2007).

12. Hansen, J. J. et al. Hereditary spastic paraplegia SPG13 is associated with a mutation in the gene encoding the mitochondrial chaperonin Hsp60. Am. J. Hum. Genet. 70, 1328-1332 (2002).

13. Magen, D. et al. Mitochondrial hsp60 chaperonopathy causes an autosomalrecessive neurodegenerative disorder linked to brain hypomyelination and leukodystrophy. Am. J. Hum. Genet. 83, 30-42 (2008).

14. Briones, P. et al. A new case of multiple mitochondrial enzyme deficiencies with decreased amount of heat shock protein 60. J. Inherit. Metab. Dis. 20, 569-577 (1997).

15. Venner, T. J. \& Gupta, R. S. Nucleotide sequence of mouse HSP60 (chaperonin, GroEL homolog) cDNA. Biochim Biophys. Acta 1087, 336-338 (1990).

16. Venner, T. J., Singh, B. \& Gupta, R. S. Nucleotide sequences and novel structural features of human and Chinese hamster hsp60 (chaperonin) gene families. DNA Cell Biol. 9, 545-552 (1990).

17. Rye, H. S. et al. Distinct actions of cis and trans ATP within the double ring of the chaperonin GroEL. Nature 388, 792-798 (1997).

18. Xu, Z., Horwich, A. L. \& Sigler, P. B. The crystal structure of the asymmetric GroEL-GroES-(ADP)7 chaperonin complex. Nature 388, 741-750 (1997).

19. Horovitz, A., Fridmann, Y., Kafri, G. \& Yifrach, O. Review: allostery in chaperonins. J. Struct. Biol. 135, 104-114 (2001).

20. Saibil, H. R., Fenton, W. A., Clare, D. K. \& Horwich, A. L. Structure and allostery of the chaperonin GroEL. J. Mol. Biol. 425, 1476-1487 (2013).

21. Levy-Rimler, G. et al. The effect of nucleotides and mitochondrial chaperonin 10 on the structure and chaperone activity of mitochondrial chaperonin 60 . Eur. J. Biochem./FEBS 268, 3465-3472 (2001).

22. Yifrach, O. \& Horovitz, A. Nested cooperativity in the ATPase activity of the oligomeric chaperonin GroEL. Biochemistry 34, 5303-5308 (1995).
23. Sameshima, T., Iizuka, R., Ueno, T. \& Funatsu, T. Denatured proteins facilitate the formation of the football-shaped GroEL-(GroES)2 complex. Biochem. J. 427, 247-254 (2010).

24. Ye, X. \& Lorimer, G. H. Substrate protein switches GroE chaperonins from asymmetric to symmetric cycling by catalyzing nucleotide exchange. Proc. Natl Acad. Sci. USA 110, E4289-E4297 (2013).

25. Yang, D., Ye, X. \& Lorimer, G. H. Symmetric GroEL:GroES2 complexes are the protein-folding functional form of the chaperonin nanomachine. Proc. Natl Acad. Sci. USA 110, E4298-E4305 (2013).

26. Nielsen, K. L. \& Cowan, N. J. A single ring is sufficient for productive chaperonin-mediated folding in vivo. Mol. Cell 2, 93-99 (1998).

27. Weiss, C., Jebara, F., Nisemblat, S. \& Azem, A. Dynamic Complexes in the Chaperonin-Mediated Protein Folding Cycle. Front. Mol. Biosci. 3, 80 (2016)

28. Nisemblat, S., Yaniv, O., Parnas, A., Frolow, F. \& Azem, A. Crystal structure of the human mitochondrial chaperonin symmetrical football complex. Proc. Natl Acad. Sci. USA 112, 6044-6049 (2015).

29. Parnas, A. et al. Identification of elements that dictate the specificity of mitochondrial Hsp60 for its co-chaperonin. PLoS ONE 7, e50318 (2012).

30. Scheres, S. H. \& Chen, S. Prevention of overfitting in cryo-EM structure determination. Nat. Methods 9, 853-854 (2012).

31. Hunt, J. F., Weaver, A. J., Landry, S. J., Gierasch, L. \& Deisenhofer, J. The crystal structure of the GroES co-chaperonin at 2.8 A resolution. Nature 379, 37-45 (1996).

32. Parnas, A. et al. The MitCHAP-60 disease is due to entropic destabilization of the human mitochondrial Hsp60 oligomer. J. Biol. Chem. 284, 28198-28203 (2009).

33. Ma, J. \& Karplus, M. The allosteric mechanism of the chaperonin GroEL: a dynamic analysis. Proc. Natl Acad. Sci. USA 95, 8502-8507 (1998).

34. Braig, K. et al. The crystal structure of the bacterial chaperonin GroEL at 2.8 A. Nature 371, 578-586, https://doi.org/10.1038/371578a0 (1994).

35. Yifrach, O. \& Horovitz, A. Allosteric control by ATP of non-folded protein binding to GroEL. J. Mol. Biol. 255, 356-361 (1996).

36. Fei, X., Yang, D., LaRonde-LeBlanc, N. \& Lorimer, G. H. Crystal structure of a GroEL-ADP complex in the relaxed allosteric state at 2.7 A resolution. Proc. Natl Acad. Sci. USA 110, E2958-E2966 (2013).

37. Fei, X., Ye, X., LaRonde, N. A. \& Lorimer, G. H. Formation and structures of GroEL:GroES2 chaperonin footballs, the protein-folding functional form. Proc. Natl Acad. Sci. USA 111, 12775-12780 (2014).

38. Gruber, R., Levitt, M. \& Horovitz, A. Sequential allosteric mechanism of ATP hydrolysis by the CCT/TRiC chaperone is revealed through Arrhenius analysis. Proc. Natl Acad. Sci. USA 114, 5189-5194 (2017)

39. Lorimer, G. H., Fei, X. \& Ye, X. The GroEL chaperonin: a protein machine with pistons driven by ATP binding and hydrolysis. Philos. Trans. $R$ Soc. Lond. B Biol. Sci. 373, https://doi.org/10.1098/rstb.2017.0179 (2018).

40. Todd, M. J., Viitanen, P. V. \& Lorimer, G. H. Dynamics of the chaperonin ATPase cycle: implications for facilitated protein folding. Science 265, 659-666 (1994).

41. Sewell, B. T. et al. A mutant chaperonin with rearranged inter-ring electrostatic contacts and temperature-sensitive dissociation. Nat. Struct. Mol. Biol. 11, 1128-1133 (2004).

42. Sot, B. et al. Ionic interactions at both inter-ring contact sites of GroEL are involved in transmission of the allosteric signal: a time-resolved infrared difference study. Protein Sci. 14, 2267-2274 (2005).

43. Bandyopadhyay, B., Mondal, T., Unger, R. \& Horovitz, A. Contact order is a determinant for the dependence of GFP folding on the chaperonin GroEL. Biophys. J. 116, 42-48 (2019).

44. Viitanen, P. V. et al. Purification of mammalian mitochondrial chaperonin 60 through in vitro reconstitution of active oligomers. Methods Enzymol. 290, 203-217 (1998).

45. Horwich, A. L. \& Fenton, W. A. Chaperonin-mediated protein folding: using a central cavity to kinetically assist polypeptide chain folding. Q Rev. Biophys. 42, 83-116 (2009)

46. Koike-Takeshita, A., Arakawa, T., Taguchi, H. \& Shimamura, T. Crystal structure of a symmetric football-shaped GroEL:GroES2-ATP14 complex determined at 3.8A reveals rearrangement between two GroEL rings. J. Mol. Biol. 426, 3634-3641 (2014).

47. Goodsell, D. S. \& Olson, A. J. Structural symmetry and protein function. Annu. Rev. Biophys. Biomol. Struct. 29, 105-153 (2000).

48. Andre, I., Strauss, C. E., Kaplan, D. B., Bradley, P. \& Baker, D. Emergence of symmetry in homooligomeric biological assemblies. Proc. Natl Acad. Sci. USA 105, 16148-16152 (2008).

49. Yan, X. et al. GroEL ring separation and exchange in the chaperonin reaction. Cell 172, 605-617 e611 (2018).

50. Farr, G. W. et al. Folding with and without encapsulation by cis- and transonly GroEL-GroES complexes. EMBO J. 22, 3220-3230 (2003).

51. Bigman, L. S. \& Horovitz, A. Reconciling the controversy regarding the functional importance of bullet- and football-shaped GroE complexes. J. Biol. Chem. 294, 13527-13529 (2019). 
52. Opatowsky, Y., Chomsky-Hecht, O., Kang, M. G., Campbell, K. P. \& Hirsch, J. A. The voltage-dependent calcium channel beta subunit contains two stable interacting domains. J. Biol. Chem. 278, 52323-52332 (2003).

53. Goloubinoff, P., Diamant, S., Weiss, C. \& Azem, A. GroES binding regulates GroEL chaperonin activity under heat shock. FEBS Lett. 407, 215-219 (1997).

54. Sokolovski, M., Bhattacherjee, A., Kessler, N., Levy, Y. \& Horovitz, A. Thermodynamic protein destabilization by GFP tagging: a case of interdomain allostery. Biophysical J. 109, 1157-1162 (2015).

55. Kabsch, W. Xds. Acta Crystallogr D. Biol. Crystallogr 66, 125-132 (2010).

56. Afonine, P. V. et al. Towards automated crystallographic structure refinement with phenix.refine. Acta Crystallogr. D. Biol. Crystallogr. 68, 352-367 (2012).

57. Emsley, P. \& Cowtan, K. Coot: model-building tools for molecular graphics. Acta Crystallogr. D. Biol. Crystallogr. 60, 2126-2132 (2004).

58. Glaeser, R. M., Typke, D., Tiemeijer, P. C., Pulokas, J. \& Cheng, A. Precise beam-tilt alignment and collimation are required to minimize the phase error associated with coma in high-resolution cryo-EM. J. Struct. Biol. 174, 1-10 (2011).

59. Suloway, C. et al. Automated molecular microscopy: the new Leginon system. J. Struct. Biol. 151, 41-60 (2005).

60. Zheng, S. Q. et al. MotionCor2: anisotropic correction of beam-induced motion for improved cryo-electron microscopy. Nat. Methods 14, 331-332 (2017).

61. Rohou, A. \& Grigorieff, N. CTFFIND4: fast and accurate defocus estimation from electron micrographs. J. Struct. Biol. 192, 216-221 (2015).

62. Roseman, A. M. FindEM - a fast, efficient program for automatic selection of particles from electron micrographs. J. Struct. Biol. 145, 91-99 (2004).

63. Lander, G. C. et al. Appion: an integrated, database-driven pipeline to facilitate EM image processing. J. Struct. Biol. 166, 95-102 (2009).

64. Scheres, S. H. RELION: implementation of a Bayesian approach to cryo-EM structure determination. J. Struct. Biol. 180, 519-530 (2012).

65. Punjani, A., Rubinstein, J. L., Fleet, D. J. \& Brubaker, M. A. cryoSPARC: algorithms for rapid unsupervised cryo-EM structure determination. Nat. Methods 14, 290-296 (2017).

66. Kucukelbir, A., Sigworth, F. J. \& Tagare, H. D. Quantifying the local resolution of cryo-EM density maps. Nat. Methods 11, 63-65 (2014).

67. Adams, P. D. et al. PHENIX: a comprehensive Python-based system for macromolecular structure solution. Acta Crystallogr. D. Biol. Crystallogr. 66, 213-221 (2010).

\section{Acknowledgements}

We acknowledge support from the United States-Israel Binational Science Foundation (grant number 2015214) to A.A. and I.U.-B. F.J. was supported by a fellowship from the Planning and Budgeting Committee of the Israel Council for Higher Education. I.U.-B. was supported in part by the Fundación Biofísica Bizkaia and the Basque Excellence Research Centre program. Negative stained data collection and initial cryo-EM screening was performed at the Icahn School of Medicine microscope facility supported by a shared instrumentation grant from the NIH (1S10RR026473). High-resolution cryo-EM data collection was performed at the Simons Electron Microscopy Center located at the New York Structural Biology Center, supported by grants from the Simons Foundation
(SF349247), NYSTAR, and the NIH National Institute of General Medical Sciences (GM103310) with additional support from Agouron Institute (F00316) and NIH (OD019994). Computing resources needed for this work were provided in part by the scientific computing facility of the Icahn School of Medicine at Mount Sinai. We thank Dr. Amnon Horovitz (Weizmann Institute of Science) for the D116/G117-eGFP mutant expression plasmid. We are grateful to Dr. George Lorimer (University of Maryland) for the algorithm to analyze structures using a cylindrical coordinate system. We also thank Dr. Igor Tascon (Instituto Biofisika) for helpful discussions.

\section{Author contributions}

Y.G.-L.L., R.M., J.A.H., and I.U.-B. performed the cryo-EM studies. F.J., M.P., and J.A.H. performed the X-ray crystallography studies and model building. F.J., M.P., S.N., A.P., O.C.-H., and A.A. performed molecular biology, protein purification, native gel experiments and functional characterization. Y.G.-L.L., F.J., A.A., J.A.H., and I.U.-B. wrote the paper. A.A., J.A.H., and I.U.-B. designed the research.

\section{Competing interests}

The authors declare no competing interests.

\section{Additional information}

Supplementary information is available for this paper at https://doi.org/10.1038/s41467020-15698-8.

Correspondence and requests for materials should be addressed to A.A., J.A.H. or I.U.-B.

Peer review information Nature Communications thanks David Libich, and other, anonymous reviewer(s) for their contribution to the peer review of this work.

Reprints and permission information is available at http://www.nature.com/reprints

Publisher's note Springer Nature remains neutral with regard to jurisdictional claims in published maps and institutional affiliations.

Open Access This article is licensed under a Creative Commons Attribution 4.0 International License, which permits use, sharing, adaptation, distribution and reproduction in any medium or format, as long as you give appropriate credit to the original author(s) and the source, provide a link to the Creative Commons license, and indicate if changes were made. The images or other third party material in this article are included in the article's Creative Commons license, unless indicated otherwise in a credit line to the material. If material is not included in the article's Creative Commons license and your intended use is not permitted by statutory regulation or exceeds the permitted use, you will need to obtain permission directly from the copyright holder. To view a copy of this license, visit http://creativecommons.org/ licenses/by/4.0/.

(C) The Author(s) 2020 